\title{
High-precision density measurements in the solar corona
}

\section{Analysis methods and results for Fe XII and Fe XIII}

\author{
P. R. Young ${ }^{1,2,3, \star}$, T. Watanabe ${ }^{4}$, H. Hara ${ }^{4}$, and J. T. Mariska ${ }^{3}$ \\ 1 Rutherford Appleton Laboratory, Chilton, Didcot, Oxfordshire OX11 0QX, UK \\ e-mail: peter.young.ctr.uk@nrl.navy.mil \\ George Mason University, 4400 University Drive, Fairfax, VA 22030, USA \\ 3 Space Science Division, Naval Research Laboratory, Washington, DC 20375, USA \\ 4 National Astronomical Observatory of Japan, National Institutes of Natural Sciences, Mitaka, Tokyo 181-8588, Japan
}

Received 6 May 2008 / Accepted 26 September 2008

\section{ABSTRACT}

\begin{abstract}
Aims. The EUV Imaging Spectrometer (EIS) instrument on board the Hinode satellite has access to some of the best coronal density diagnostics, and the high sensitivity of the instrument now allows electron number density, $N_{\mathrm{e}}$, measurements to an unprecedented precision of up to $\pm 5 \%$ in active regions. This paper gives a thorough overview of data analysis issues for the best diagnostics of Fe XII and Fe XIII and assesses the accuracy of the measurements.

Methods. Two density diagnostics each from Fe XII $(\lambda 186.88 / \lambda 195.12$ and $\lambda 196.64 / \lambda 195.12)$ and Fe XIII $(\lambda 196.54 / \lambda 202.04$ and $\lambda 203.82 / \lambda 202.04)$ are analysed in two active region datasets from 2007 May 3 and 6 that yield densities in the range $8.5 \leq$ $\log \left(N_{\mathrm{e}} / \mathrm{cm}^{-3}\right) \leq 11.0$. The densities are derived using v5.2 of the CHIANTI atomic database. Blending, line fitting, and instrumental issues are discussed, and line fit parameters presented.

Results. The Fe XII and Fe XIII diagnostics show broadly the same trend in density across the active region, consistent with their similar temperatures of formation. However, the high precision of the EIS measurements demonstrates significant discrepancies of up to 0.5 dex in derived $\log N_{\mathrm{e}}$ values, with Fe XII always giving higher densities than Fe XIII. The discrepancies may partly be due to real physical differences between the emitting regions of the two plasmas, but the dominant factor lies in the atomic models of the two ions. Two specific problems are identified for Fe XII $\lambda 196.64$ and Fe XIII $\lambda 203.82$ : the former is found to be underestimated in strength by the CHIANTI atomic model, while the high-density limit of the $\lambda 203.82 / \lambda 202.04$ ratio appears to be inaccurate in the CHIANTI atomic model. The small grating tilt of the EIS instrument is found to be very significant when deriving densities from emission lines separated by more than a few angstroms. Revised wavelengths of $196.518 \pm 0.003 \AA$ and $196.647 \pm 0.003 \AA$ are suggested for the Fe XIII $\lambda 196.54$ and Fe XII $\lambda 196.64$ lines, respectively.
\end{abstract}

Key words. Sun: corona - Sun: UV radiation

\section{Introduction}

Density is a fundamental plasma parameter that is accessible to astrophysical spectroscopists through the density diagnostic - a ratio of two emission lines that is sensitive to the electron density of the emitting plasma. High accuracy density measurements are of direct importance for constraining energy and pressure balance within coronal structures, and also of indirect importance to the measure of the 'filling factor' of the plasma, i.e., the fraction (by volume) of the observed plasma structure that is actually emitting radiation. The latter is of significance when the spatial resolution of the telescope is not sufficient to resolve the physical structures of interest, as is often the case even for our nearest star. The power of emission line density diagnostics lies in the fact that, when the plasma is optically thin, the derived density depends purely on the atomic data for the emitting ion and is free of any physical assumptions about the volume, abundances or temperature structure within the plasma.

Density diagnostics for use in both analyses of stellar coronae and nebulae have been described and exploited for many years. For the solar corona, reviews of early work are provided

* Present address: Space Science Division, Naval Research Laboratory, Washington, DC 20375, USA. by Dere \& Mason (1981) and Mariska (1992). Steady improvements in the accuracy of atomic data calculations and access to high resolution, calibrated ultraviolet spectra from space-borne instruments have led to a number of detailed studies of density diagnostics from both individual ions and sequences of ions, e.g., Brickhouse et al. (1995), Laming et al. (1997) and Young et al. (1998).

A particular advance has been the routine access to most of the wavelength range $150-1600 \AA$ by the CDS and SUMER instruments on board the $\mathrm{SOHO}$ spacecraft for over a 12 year period. A wide range of density diagnostics from many different ions has been exploited (e.g., Doschek et al. 1997; Laming et al. 1997; Young et al. 1999; Del Zanna \& Mason 2003), however the density measurements from these two instruments are generally accurate only to the $30-40 \%$ level at best and often it is necessary to bin multiple spatial pixels or exposures to obtain a useful signal. This is because the most commonly-used diagnostics generally involve only weak-to-medium strength emission lines and/or the diagnostics themselves do not have high sensitivity to density.

The best density diagnostics at typical coronal temperatures (1-3 MK) belong to the set of iron ions Fe IX-XV. They arise because of the complex atomic structures of these ions which 
lead to a number of metastable levels that are the source of the density sensitivity of the emission line ratios (e.g., Sect. 6.3.1 of Dere \& Mason 1981). The density diagnostics of these iron ions are mostly found below $300 \AA$, a wavelength region not well covered by the $\mathrm{SOHO}$ spectrometers.

The EUV Imaging Spectromer (EIS) on board the Hinode satellite - launched during 2006 September - observes two wavelength ranges below $300 \AA$ and, for the first time, scientists have routine access to high spectral and spatial resolution data in this region. A key factor is the high sensitivity of the instrument which is enabled by a simple optical design that incorporates multilayer coatings (Culhane et al. 2007). The sensitivity is particularly high in the range 185-205 $\AA$ where are found excellent density diagnostics from Fe XII and Fe XIII. As demonstrated in the following sections, diagnostics from these ions yield density measurements with an unprecedented precision of up to $5 \%$ in individual spatial pixels at exposure times of $30 \mathrm{~s}$ or less - a vast improvement over earlier instruments.

The diagnostic potential of Fe XII and Fe XIII has been recognised for many years. The first sophisticated atomic calculation for Fe XII was performed by Flower (1977) and a number of UV density diagnostics have been studied (e.g., Feldman et al. 1983; Tayal et al. 1991), but for many years there were large discrepancies between predictions from atomic data models and measured line intensities which were summarised in Binello et al. (2001). Finally the large $R$-matrix calculation performed by Storey et al. (2005) resolved many of the problems (Del Zanna \& Mason 2005). The three emission lines used in the present work $(\lambda 186.88, \lambda 195.12$ and $\lambda 196.64)$ were shown by Del Zanna \& Mason (2005) to give good agreement with other line ratios from Fe XII, but each line is affected by line blending. This will be discussed in detail during the course of the present work.

For Fe XIII, Flower \& Nussbaumer (1974) calculated atomic data and produced line ratio calculations that yielded reasonably consistent density measurements from a quiet Sun spectrum. Detailed comparisons of the more recent atomic data models of Fe XIII in the CHIANTI atomic database (Dere et al. 1997) with spectra from the SERTS rocket flight experiments (Neupert et al. 1992) have been performed by Young et al. (1998) and Landi (2002). In particular, the latter work found that the $\lambda 196.54 / \lambda 202.04$ and $\lambda 203.82 / \lambda 202.04$ ratios used in the present work give density estimates in good agreement with each other and also with other ions formed at similar temperatures.

Early density results from EIS obtained using the Fe XII and Fe XIII diagnostics have been presented in Brooks et al. (2007), Watanabe et al. (2007), Doschek et al. (2007a), Doschek et al. (2007b) and Dere et al. (2007). In particular we highlight the density maps shown in Doschek et al. (2007b) that demonstrate the high quality of the EIS density measurements, and the density comparisons shown in Watanabe et al. (2007) that show excellent agreement between four different iron ion density diagnostics.

In this paper density measurements from two diagnostics each of the Fe XII and Fe XIII ions are presented from two active region data sets. A careful discussion of line fitting and blending issues is given, and an estimate of the accuracy of the density measurements is made. A following paper will use the derived densities to estimate column depths and filling factors for the two ions.

\section{Instrument details}

The EUV Imaging Spectrometer (EIS) on Hinode observes two wavelength ranges: $170-212 \AA$ and 246-292 $\AA$. The instrument is described in detail by Korendyke et al. (2006) and Culhane et al. (2007). The key optical elements are: a parabolic primary mirror for focussing incoming radiation; a uniform line-spaced grating for dispersing the radiation and two back-thinned, EUV sensitive CCDs for detecting the radiation. Two aluminium filters lie on the optical path for blocking visible radiation, and a slit-slot assembly is situated between the mirror and grating containing four different-sized slits for selecting the spatial region to be sent to the spectromer. The optical surfaces of both the mirror and grating are divided in two and coated with multilayer coatings optimised for the two wavelength bands. Of the four entrance slits, two are available for detailed spectroscopy work with projected widths at the Sun of $1^{\prime \prime}$ and $2^{\prime \prime}$. Pixel sizes on the detector are $1^{\prime \prime}$ (spatial dimension) and $0.0223 \AA$ (spectral dimension). For single exposures the maximum field-of-view in Solar-Y is $512^{\prime \prime}$.

For density measurements, the absolute radiometric calibration of the EIS instrument is not important, only the relative calibration between the wavelengths of the lines. The key uncertainty is whether the relative reflectivities from the mirror and grating between two wavelengths deviate from the values contained in the calibration pipeline. There are two components: the uncertainty due to variations in reflectivity across the optical surfaces; and a systematic error in the reflectivity curves due to some change in the instrument between the laboratory calibration measurements and launch. For the former, measurements performed on flight-quality mirrors and gratings by Seely et al. (2004) revealed that, when a beamline is scanned across the optical surfaces, the reflectivity as a function of wavelength is found to vary. Seely et al. (2004) averaged the resulting reflectivity curves and the standard deviations of the data points for the wavelengths considered here range from 3 to $8 \%$. In orbit the optical surfaces are fully illuminated which will serve to average out the variations and so these standard deviation values will be over-estimates of the actual uncertainty in the relative calibration. In addition, these percentage errors are for the absolute reflectivity - the standard deviation for the relative reflectivities between two lines close in wavelength (e.g., the Fe XIII $\lambda 202.04$ and $\lambda 203.82$ ) will likely be significantly less. For this reason we do not include any estimate of the relative calibration uncertainty in the error budget for derived densities. The error bars for the density measurements presented here are thus underestimates, but we believe the uncertainties in the relative calibration are comparable to or smaller than these errors and would not significantly impact the conclusions of this work. Of greater concern for the present work is whether there is a systematic error in the shape of the multilayer reflectivity curves used in the EIS calibration. This can be investigated through the study of emission line ratios that are insensitive to the plasma conditions - an example being the Fe XII ratios considered in Appendix B. The question of systematic errors is considered in the later sections when the density results are presented.

The basic unit of observation for EIS is a raster which is a consecutive set of exposures that either scan a spatial region (from solar-west to solar-east) on the Sun, or are fixed relative to the Sun's surface ("sit-and-stare" observations). A study is defined as a collection of one or more rasters that is designed to perform a particular science task. Each study and raster has a unique 20 character acronym that is used to identify it for science planning purposes. 
Table 1. Transition information and CHIANTI wavelengths for the density diagnostics.

\begin{tabular}{llll}
\hline \hline Ion & Wavelength/ $\AA$ & Transition & $\mathrm{EA}^{a} / \mathrm{cm}^{-2}$ \\
\hline Fe XII & 186.854 & $3 \mathrm{~s}^{2} 3 \mathrm{p}^{3}{ }^{2} \mathrm{D}_{3 / 2}-3 \mathrm{~s}^{2} 3 \mathrm{p}^{2}\left({ }^{3} \mathrm{P}\right) 3 \mathrm{~d}^{2} \mathrm{~F}_{5 / 2}$ & 0.114 \\
& 186.887 & $3 \mathrm{~s}^{2} 3 \mathrm{p}^{3}{ }^{2} \mathrm{D}_{5 / 2}-3 \mathrm{~s}^{2} 3 \mathrm{p}^{2}\left({ }^{3} \mathrm{P}\right) 3 \mathrm{~d}^{2} \mathrm{~F}_{7 / 2}$ & 0.115 \\
& 195.119 & $3 \mathrm{~s}^{2} 3 \mathrm{p}^{3}{ }^{4} \mathrm{~S}_{3 / 2}-3 \mathrm{~s}^{2} 3 \mathrm{p}^{2}\left({ }^{3} \mathrm{P}\right) 3 \mathrm{~d}^{4} \mathrm{P}_{5 / 2}$ & 0.303 \\
& 196.640 & $3 \mathrm{~s}^{2} 3 \mathrm{p}^{3}{ }^{2} \mathrm{D}_{5 / 2}-3 \mathrm{~s} 3 \mathrm{p}^{3}\left({ }^{1} \mathrm{D}\right) 3 \mathrm{~d}^{2} \mathrm{D}_{5 / 2}$ & 0.301 \\
Fe XIII & 196.540 & $3 \mathrm{~s}^{2} 3 \mathrm{p}^{2}{ }^{1} \mathrm{D}_{2}-3 \mathrm{~s}^{2} 3 \mathrm{p} 3 \mathrm{~d}^{1} \mathrm{~F}_{3}$ & 0.302 \\
& 202.044 & $3 \mathrm{~s}^{2} 3 \mathrm{p}^{2}{ }^{3} \mathrm{P}_{0}-3 \mathrm{~s}^{2} 3 \mathrm{p} 3 \mathrm{~d}^{3} \mathrm{P}_{1}$ & 0.084 \\
& 203.797 & $3 \mathrm{~s}^{2} 3 \mathrm{p}^{2}{ }^{3} \mathrm{P}_{2}-3 \mathrm{~s}^{2} 3 \mathrm{p} 3 \mathrm{~d}^{3} \mathrm{D}_{2}$ & 0.044 \\
& 203.828 & $3 \mathrm{~s}^{2} 3 \mathrm{p}^{2}{ }^{3} \mathrm{P}_{2}-3 \mathrm{~s}^{2} 3 \mathrm{p} 3 \mathrm{~d}^{3} \mathrm{D}_{3}$ & 0.044 \\
\hline
\end{tabular}

${ }^{a}$ Effective area values.

On account of data download volume restrictions, complete CCD images from each EIS exposure are not routinely transmitted to Earth. A key part of the raster definition process is thus to define "wavelength windows" on the detector for selecting particular emission lines and/or a reduced spatial coverage in the Solar-Y direction. The widths of the windows need to be chosen carefully by the scientist so that accurate fitting of the emission line profiles will be possible. Typically window widths are chosen to be $24-32$ pixels $(0.54-0.71 \AA$ ) but for some of the emission lines considered here wider windows are necessary, and this will be discussed in the following sections.

\section{Atomic data}

To convert a measured line ratio value to a density value it is necessary to have a model for the atomic processes within the ion that predicts the relative strengths of the emission lines as a function of density. Here we use version 5.2 of the CHIANTI atomic database (Landi et al. 2006; Dere et al. 1997) which contains atomic data and analysis software for calculating line ratios as a function of density. For Fe XII the data consist of energy levels and radiative decay rates from Del Zanna \& Mason (2005), electron collision strengths from Storey et al. (2005), and proton collision rate coefficients from Landman (1978). For Fe XIII energy levels are from Penn \& Kuhn (1994), Jupen et al. (1993) and version 1.0 of the NIST database; radiative decay rates are from Young (2004), electron collision strengths from Gupta \& Tayal (1998), and proton collision rate coefficients from Landman (1975). During the course of the present work, the CHIANTI team found an error in the CHIANTI electron collision strength file for Fe XIII, which actually contained older data from Fawcett \& Mason (1989) rather than the data of Gupta $\&$ Tayal (1998) that were supposed to have been added in version 3 of the database (Dere et al. 2001). With the Gupta \& Tayal (1998) dataset Fe XIII yields significantly different densities (Landi 2002).

The atomic transitions of the Fe XII and Fe XIII density diagnostic lines considered here are given in Table 1, and plots showing how the ratios are predicted to vary with density are shown in Fig. 3 of Young et al. (2007b). The diagnostics themselves are the three recommended by Young et al. (2007b) - FeXII $\lambda 196.64 / \lambda 195.12$, and FeXIII $\lambda 196.54 / \lambda 202.04$ and $\lambda 203.82 / \lambda 202.04$ - together with Fe XII $\lambda 186.88 / \lambda 195.12$ which is comparable to $\lambda 196.64 / \lambda 195.12$ but concern was expressed by Young et al. (2007b) over the blending line S XI $\lambda 186.84$. This issue will be discussed further in Sect. 5.1. Between them, these four density diagnostics are the best available in the EIS wavelength bands. The Fe XII diagnostics benefit from the high instrument sensitivity at $195.12 \AA$ which leads to accurate measurements of the line ratios. The Fe XII diagnostics also benefit from a wide sensitivity to density from $10^{8}$ to $10^{12} \mathrm{~cm}^{-3}$ that arises through the shift of population from the ground ${ }^{4} \mathrm{~S}$ term to first the ${ }^{2} \mathrm{D}$ term and then the ${ }^{2} \mathrm{P}$ term as density increases over this range. The EIS sensitivity for Fe XIII $\lambda 202.04$ and $\lambda 203.82$ is lower than for the Fe XII lines (Table 1), but the value of the diagnostics lies in the strong sensitivity of the lines to density. E.g., the $\lambda 203.82 / \lambda 202.04$ ratio varies by a factor 43 from $10^{8}$ to $10^{10} \mathrm{~cm}^{-3}$, whereas Fe XII $\lambda 196.64 / \lambda 195.12$ varies by a factor 8 . This high sensitivity means that the error bars on a measured ratio translate to relatively small errors on the derived density.

A more recent set of collision data for Fe XIII than that in CHIANTI was published by Aggarwal \& Keenan (2005), and these have been compared with the v5.2 CHIANTI Fe XIII model and SERTS rocket flight observations in Keenan et al. (2007). The Fe XIII model used by Keenan et al. (2007) consisted of collision data from Aggarwal \& Keenan (2005) and radiative data from Aggarwal \& Keenan (2004). The agreement with the CHIANTI model was found to be very good (although this was the CHIANTI model containing the Fawcett \& Mason 1989, data rather than the model used here). Figure 1 compares the theoretical ratios from our revised CHIANTI model and Keenan et al. (2007) for the two Fe XIII ratios. In both cases, for a given ratio value, the Aggarwal \& Keenan (2005) data yield lower densities by around 0.1-0.2 dex. The high-density limits from Keenan et al. (2007) are higher than those from CHIANTI which has a significant impact on derived results from high density plasmas. These issues will be discussed further in Sects. 6 and 7. Keenan et al. (2007) presented high resolution spectra from the SERTS95 rocket flight (Brosius et al. 1998) where they were able to resolve the two components of the Fe XIII $\lambda 203.82$ blend, i.e., the lines at 203.79 and $203.83 \AA$. This is not possible with the lower resolution EIS spectra. Keenan et al. (2007) found that the $\lambda 203.79 / \lambda 203.83$ ratio was significantly different from theoretical predictions, with an observed value of 0.20 and a theoretical value of 0.32 . This raises concerns about the suitability of using the blended $\lambda 203.82$ line as part of a density diagnostic. We note, however, that summing the $\lambda 203.79$ and $\lambda 203.83$ intensities from the SERTS-95 spectrum and forming a density diagnostic with 2202.04 , the derived density (using the Keenan et al. 2007, model) is $\log N_{\mathrm{e}}=9.1$ which is consistent with the other Fe XIII densities presented in Table 9 of Keenan et al. (2007). We note the line widths measured by Keenan et al. (2007) for $\lambda 203.79$ and $\lambda 203.83$ are significantly different which is not expected from two lines of the same ion, and this may partly be responsible for the discrepancy between theory and observation for their intensity ratio. For the remainder of this work 


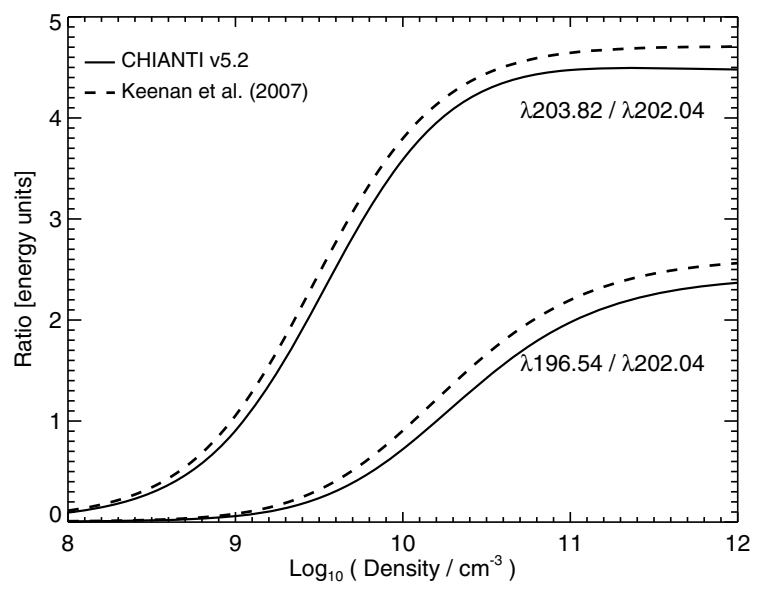

Fig. 1. Theoretical variation of emission line ratios versus electron density for Fe XIII $\lambda 203.82 / \lambda 202.04$ and $\lambda 196.54 / \lambda 202.04$. The solid lines show the results from the modified CHIANTI v5.2 model while the dashed lines show results from the Fe XIII model of Keenan et al. (2007).

we will concentrate on the density results from the modified Fe XIII CHIANTI model as CHIANTI is widely used amongst the solar physics community.

The standard CHIANTI models for Fe XII and Fe XIII include electron excitation/de-excitation, spontaneous radiative decay and proton excitation/de-excitation. Further processes that can be included are photo-excitation and stimulated emission which can be significant in low density conditions when electron collisions are less frequent. The method for including these latter processes in CHIANTI is described in Young et al. (2003), and examples of the effects of the processes on density diagnostics are provided in Flower \& Pineau des Forêts (1973) and Young \& Esser (1999a,b). For the solar case, the radiation field to be considered is the photospheric continuum which is modelled in CHIANTI as a black-body. For Fe XII, such a radiation field has no effect on the density sensitive ratios considered in this work. For Fe XIII there is no significant effect on $\lambda 196.64 / \lambda 202.04$, but there is an effect for $\lambda 203.82 / \lambda 202.04$.

Figure 2 compares the predicted variation of Fe XIII $\lambda 203.82 / \lambda 202.04$ with density for two cases: when there is no incident radiation field (solid line) and when the Fe XIII emitting plasma is located $10^{\prime \prime}$ above the solar surface (dashed line). The radiation field serves to push the curve towards lower densities. A measured intensity ratio will thus yield a lower density when the radiation field is taken into account: for $\log N_{\mathrm{e}} \approx 9$ the reduction is about $0.05 \mathrm{dex}$. The change becomes less for higher densities, being negligible above $\log N_{\mathrm{e}}=10$. The location of the Fe XIII emitting plasma above the photosphere is also a factor, with greater distances leading to a smaller effect. For the present work the effects of an incident radiation field will not be considered any further since the magnitude of the effect is small compared to some of the discrepancies discussed later, and also it is not possible to make a general statement about the location of the Fe XIII emitting plasma in the data sets considered.

The present work is focussed on density diagnostics and so wavelengths and velocity measurements are not important. For all reference wavelengths quoted in the text, we use the the values from v.5.2 of the CHIANTI database (Landi et al. 2006). In particular we note the revised Fe XII wavelengths of Del Zanna \& Mason (2005) which are included in CHIANTI and are valuable for interpreting the Fe XII $\lambda 195.12$ line (Sect. 5.2). A by-product

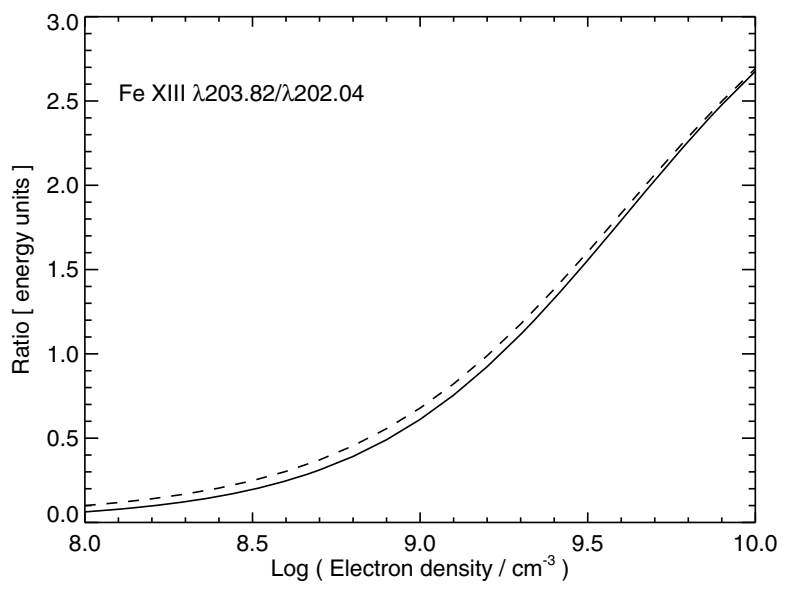

Fig. 2. The theoretical variation of the Fe XIII $\lambda 203.82 / \lambda 202.04$ ratio as a function of density, as derived from CHIANTI. Two cases are shown: the curve calculated assuming no incident radiation field (solid line), and the curve calculated assuming the Fe XIII emitting plasma is located $10^{\prime \prime}$ above a photosphere of temperature $6000 \mathrm{~K}$.

of the Gaussian fits to the emission line profiles is line centroid measurements for each line, and these can be used to assess the accuracy of the CHIANTI wavelengths (Sect. 8).

\section{Data calibration and error bars}

The EIS data presented here were all calibrated from the level-0 FITS files using the EIS_PREP routine available in the Solarsoft ${ }^{1}$ IDL distribution. In addition to converting the measured CCD signal into calibrated intensity units, a key part of EIS_PREP is to flag bad data points. These can arise through pixel saturation, cosmic ray hits, or simply defective pixels on the CCD. In this work all such pixels were marked as "missing" data and not included in the data analysis. The central outputs of EIS_PREP are two level-1 FITS files, one containing calibrated intensities at each pixel, and the other containing error bars on these intensities. The steps performed by the current version of EIS_PREP at the time of this analysis are described below in detail.

The first step of EIS_PREP is to flag any saturated data. The EIS CCDs have a 14 bit dynamic range and so saturation occurs at 16383 data numbers (DN). All such pixels are flagged as missing as described above.

In the raw data, the spectra are found to sit on a background of around $500 \mathrm{DN}$ that arises principally from the CCD bias, and secondly from the CCD dark current. It is not possible to estimate the CCD bias level directly for EIS data, so the bias and dark current levels are estimated directly from the science data as follows. For each 3D data window $2 \%$ of the detector pixels are isolated that have the lowest $\mathrm{DN}$ values. The median $\mathrm{DN}$ value of these $2 \%$ pixels is then set to be the background level and it is subtracted from the DN values of each pixel.

Anomalously bright pixels are found on the EIS CCD images that arise from "hot pixels", "warm pixels" and cosmic rays. Both hot pixels and warm pixels are single pixels that have anomalously high DN values. A hot pixel is defined to be one that yields 25000 electrons pixel ${ }^{-1} \mathrm{~s}^{-1}$ at room temperature

\footnotetext{
1 Solarsoft is a set of integrated software libraries, databases, and system utilities that provide a common programming and data analysis environment for Solar Physics. It is available at http://www. Imsal. com/solarsoft.
} 
(a specification from the CCD manufacturer). Pixels that fall below this threshold but are still clearly identified as being anomalous when inspecting the data are referred to as "warm" pixels. Maps of the locations of hot pixels are generated by the EIS team every 2-4 weeks following inspection of $100 \mathrm{~s}$ dark exposures and they are stored in Solarsoft. The hot pixel map that is closest in time to the science observation is used by EIS_PREP to mark the hot pixels as missing data.

Before removing warm pixels and cosmic rays, the next step for EIS_PREP is to flag the pixels affected by dust on the CCD. Several small pieces of dust accumulated on the CCD before launch and are found to completely block the solar signal on the CCD at their locations. They are fixed in position and cover less than $0.1 \%$ of the $\mathrm{CCD}$, however two of the pieces do affect the strong lines Fe XI $\lambda \lambda 188.23,188.30$ and Fe XII $\lambda 193.51$ such that the lines can not be used over 15-30" spatial ranges in solar-Y.

At the time of performing the present analysis, EIS_PREP did not specifically remove warm pixels, but many of these were removed naturally by the cosmic ray removal routine, EIS_DESPIKE. This latter is a wrapper routine that calls NEW_SPIKE, a routine developed for removing cosmic rays from $\mathrm{SOHO/Coronal} \mathrm{Diagnostic} \mathrm{Spectrometer} \mathrm{(CDS)} \mathrm{data} \mathrm{sets}$ (Thompson et al. 1998; Pike \& Harrison 2000). For CDS data processing it was typical for not only the identified CCD pixels to be flagged, but also the nearest-neighbour pixels on the CCD. This is because there is often residual signal from the cosmic ray next to the brightest pixels. EIS sees significantly less cosmic rays than CDS apart from during the $\approx 5$ min passes through the South Atlantic Anomaly, and the most useful function of EIS_DESPIKE is actually to flag warm pixels. Since warm pixels are only single pixel events, then the nearestneighbour option is switched off for EIS. It is to be noted that the NEW_SPIKE routine was designed to be cautious when removing cosmic rays from line profiles thus many weak warm pixels found within spectral lines are not removed, artificially enhancing the emission line intensities at these locations. For the 2007 May data sets analysed in the present work only around $2 \%$ of the CCD pixels are warm pixels and so this is not a significant problem.

The final step of EIS_PREP is to convert DN values into intensities in units erg $\mathrm{cm}^{-2} \mathrm{~s}^{-1} \mathrm{sr}^{-1} \AA^{-1}$. The errors on the intensities are computed assuming photon statistics together with an error estimate of the dark current of $2.5 \mathrm{DN}$.

\subsection{CCD spatial offsets}

A further instrumental effect accounted for in the present work is the spatial offsets between different wavelengths. Spatial offsets between images formed from lines on the two different EIS CCDs have been discussed by Young et al. (2007a). The emission lines discussed here are exclusively from the short wavelength band and so these latter spatial offsets do not apply. However, the spectra on the short wavelength (SW) CCD are slightly tilted relative to the CCD's axes such that images from short wavelength lines are marginally higher on the CCD than long wavelength lines. The effect is due to a slight misalignment of the EIS grating relative to the CCD and which we refer to here as the "grating tilt". Note that this is not to be confused with the "slit tilt" (e.g., Mariska et al. 2007) which is a misalignment of the EIS slits relative to the CCD. This latter effect results in slit images being slightly tilted on the detector, but does not result in image misalignment between lines at different wavelengths.
The grating tilt is discussed in more detail in Appendix $\mathrm{C}$ where the gradient of the dispersion axis on the CCD is found to be -0.0792 pixels/A for the EIS SW band. Despite this small value, the effect on line ratios for lines separated by several angstroms can be significant as demonstrated in Appendix C. For all of the density diagnostic lines considered here except Fe XII $\lambda 195.12$ the 2D intensity arrays that arise after Gaussian fitting has been performed have been adjusted to account for the spatial offsets. The intensities have been modified according to Eq. (C.1), and the intensity errors have also been modified accordingly.

\section{Line fitting - method and blending issues}

In the following sections we describe the methods used to automatically fit the density diagnostic emission lines with Gaussians. Firstly, though, we must ask two questions: is it necessary to fit Gaussians to the data? and is the Gaussian an appropriate function to use? For density diagnostic work only the line intensity is needed, so it is simply necessary to sum up the intensity from the wavelength bins containing the emission lines without the need to resort to a model for the line profile. However, as will be seen, some of the lines are blended with other lines and so it is essential to have a model for the line profile in order to resolve the blending components. Fitting the lines with Gaussians yields the line centroid and line width in addition to the intensity, which thus allow the density to be related to velocity and nonthermal broadening parameters of the solar plasma, providing a much more powerful data set for scientific analysis. The widths and velocities are also valuable for assessing the accuracy of the line fitting as will be demonstrated in Sect. 8.

As to the appropriateness of Gaussian fits, in normal conditions the EIS line profiles are found to be very well represented by a Gaussian function. In particular, no evidence is found for asymmetries or enhanced wings in the line spread function. However, non-Gaussian profiles due to real solar events are seen often with EIS and examples are given in Imada et al. (2007) and Chifor et al. (2008). Typically such events are isolated and can be readily identified following an automatic Gaussian fit to the data, as they will have anomalous line widths and/or velocities. For the data analysed in the present work there is no evidence for any significant deviations from Gaussian profiles.

The high quality of the EIS data mean that the lines considered here can be automatically fit with Gaussians at virtually every spatial pixel in the EIS images. However, care has to be taken in applying automatic fitting routines due to the influence of both nearby and blending emission lines. In this section we go through each line and highlight blending and fitting issues. A key point is that the fits must be accurate and robust for the wide range of conditions encountered in the solar atmosphere. The discussion in this section builds on the initial overview of the lines presented in Young et al. (2007b).

To fit Gaussians to the EIS lines the MPFIT procedures of Markwardt ${ }^{2}$ have been implemented to automatically fit the calibrated line intensities from the spectral line windows output by the IDL routine EIS_GETWINDATA. The spectrum from each image pixel is fitted, with data points weighted with the $1 \sigma$ error bars calculated by EIS_PREP. The routine developed by Young for fitting single Gaussians to the spectral lines is called EIS_AUTO_FIT and is available in the Solarsoft IDL distribution. For some of the spectral features discussed below it was

$\overline{{ }^{2} \text { http://astrog.physics.wisc.edu/ craigm/idl/idl.html }}$ 


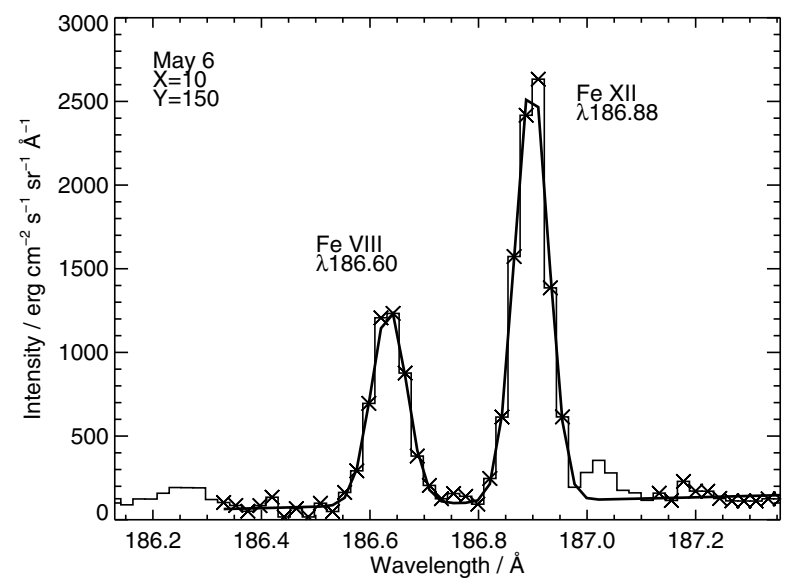

Fig. 3. An example double-Gaussian fit to the Fe VIII $\lambda 186.60$ and Fe XII $\lambda 186.88$ lines from the May 6 data set. The chosen spatial pixel is indicated. The data are plotted as a thin line and the fit with a thick line. The wavelength pixels used in the fitting are indicated by crosses. Note that the weak line on the long wavelength side of Fe XII $\lambda 186.88$ has not been included in the fit.

necessary to perform multiple Gaussian fits and custom versions of EIS_AUTO_FIT were created for these.

In the sections that follow the reference wavelengths of emission lines (denoted by, e.g., $\lambda 195.12$ ) are taken from v5.2 of the CHIANTI atomic database. Wavelengths are given to two significant figures, except where greater accuracy is required. Two of the density diagnostic lines are actually self-blends of two transitions from the ions and we use $\lambda 186.88$ to denote the blend of Fe XII $\lambda 186.854$ and $\lambda 186.887$, and $\lambda 203.82$ to denote the blend of Fe XIII $\lambda 203.797$ and $\lambda 203.828$.

\subsection{FeXII 186.88}

The emission line at $186.88 \AA$ is generally strong and wellresolved in active regions (Fig. 3) and it largely comprises of two Fe XII transitions with CHIANTI wavelengths of 186.854 and $186.887 \AA$. S XI $\lambda 186.839$ is a known blend and its contribution can be assessed from the $\lambda 188.617$ or $\lambda 191.266$ transitions which are related by branching ratios as they arise from the same upper level $\left(2 \mathrm{~s} 2 \mathrm{p}^{3}{ }^{3} \mathrm{~S}_{1}\right)$. From CHIANTI the $\lambda 186.84, \lambda 188.68$ and $\lambda 191.27$ intensities lie in the ratios $0.195: 0.580: 1.0$. In the data sets discussed in this work $\lambda 191.27$ is not observed, but $\lambda 188.68$ lies in the window containing the strong Fe XI lines at 188.22 and $188.30 \AA$ (Fig. 4).

To demonstrate the contribution of S XI to the Fe XII $\lambda 186.88$ line the intensity of S XI $\lambda 188.68$ in the May 3 and May 6 data sets considered in Sects. 6 and 7 has been measured in the Fe XI $\lambda 188.22$ window. Figure 4 shows the $\lambda 188.22$ wavelength window, where a total of six emission lines can be seen. A six Gaussian fit was applied and, to ensure robust fits, each line was assumed to have the same width. Otherwise amplitudes and centroids were free to vary. In both the May 3 and May 6 active region datasets, the six Gaussian fits were generally accurate. A 35 pixel high region on the detector is affected by the presence of a dust particle in the May 6 data set and this prevents accurate fits to the six lines in this region - hence the gap in the data points in the right panel of Fig. 5. There are also isolated pixels where poor fits are found, typically due to missing pixels or weak lines.

With the intensity of S XI $\lambda 188.68$ measured, one can estimate the intensity of S XI $\lambda 186.84$ by simply multiplying by the

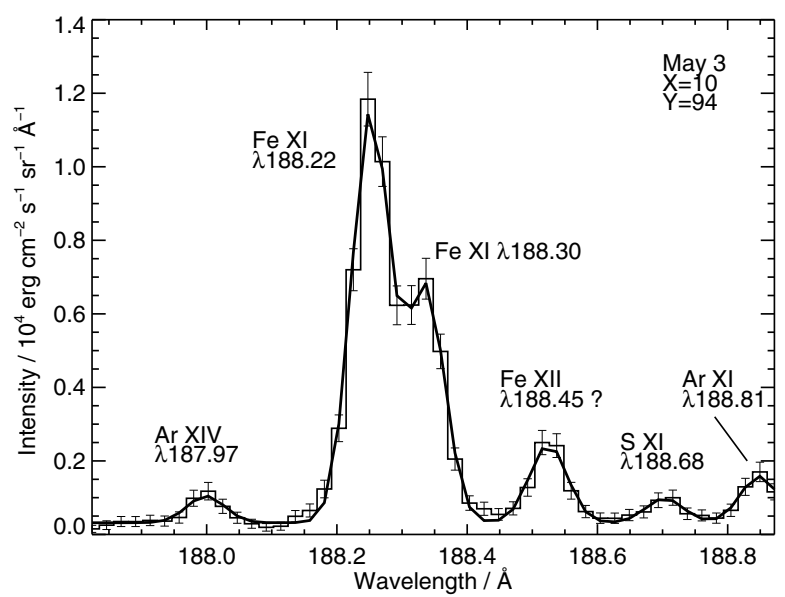

Fig. 4. Spectrum from a single spatial pixel in the May 3 dataset showing the wavelength window used to obtain the Fe XI $\lambda \lambda 188.22,188.30$ feature. A six Gaussian fit to all of the lines in the window is shown. The weak S XI $\lambda 188.68$ line used to estimate the contribution of S XI to Fe XII $\lambda 186.88$ is indicated.

factor 0.34 . This derived intensity can then be compared with the measured S XI-Fe XII feature at $186.88 \AA$ A. For the two data columns in the May 3 and May 6 datasets analysed in Sects. 6 and 7 , the results are shown in Fig. 5. The key point to note is that the majority of the pixels show a S XI contribution of $\leq 5 \%$. Where the electron density is high in the May 3 data (pixels $50-170$, see Fig. 17) the S XI contribution is around $2 \%$ as the Fe XII lines are more sensitive to higher densities than the $\mathrm{S}$ XI line.

The contribution of the S XI line at the 5\% level would have the most effect on the Fe XII densities where the Fe XII ratio is most sensitive, i.e., around $\log N_{\mathrm{e}}=10.5$. Using CHIANTI we find the Fe XII density is reduced by 0.08 dex. At $\log N_{\mathrm{e}}=9.0$ the density is reduced by 0.05 dex. We also note that Brown et al. (2008) list a Ni XIV line as possibly blending with S XI $\lambda 188.68$, potentially compromising the use of the SXI line for estimating the $\lambda 186.84$ contribution. Together with the fact that S XI $\lambda 188.68$ is a weak line that is difficult to measure, we have decided not to correct Fe XII $\lambda 186.88$ for the S XI contribution in the present analysis.

For fitting the $186.88 \AA$ feature, it is necessary to consider nearby lines that are not directly blended but can affect line fitting. Fe VIII $\lambda 186.60$ is not a blend, but sufficiently close that it can affect background estimates when fitting $\lambda 186.88$. For this work both lines have been fitted simultaneously with two independent Gaussians. A weak line is found in the long wavelength wing of $\lambda 186.88$ at $186.98 \AA$ which has been suggested to be a Ni XI transition (Brown et al. 2008). Checks on the May 3 and May 6 data sets have demonstrated that this line is always weak relative to $\lambda 186.88$, at most around $20 \%$ of the intensity. Adding an extra Gaussian to the fit to account for this line often works well, however there are a number of pixels that yield poor fits if there are missing data or weak lines. For the present work it was decided to ignore the Ni XI line by omitting the pixels at this wavelength - an example can be seen in Fig. 3. This method leads to much more robust fits while still yielding accurate estimates of the $\lambda 186.88$ intensity. 

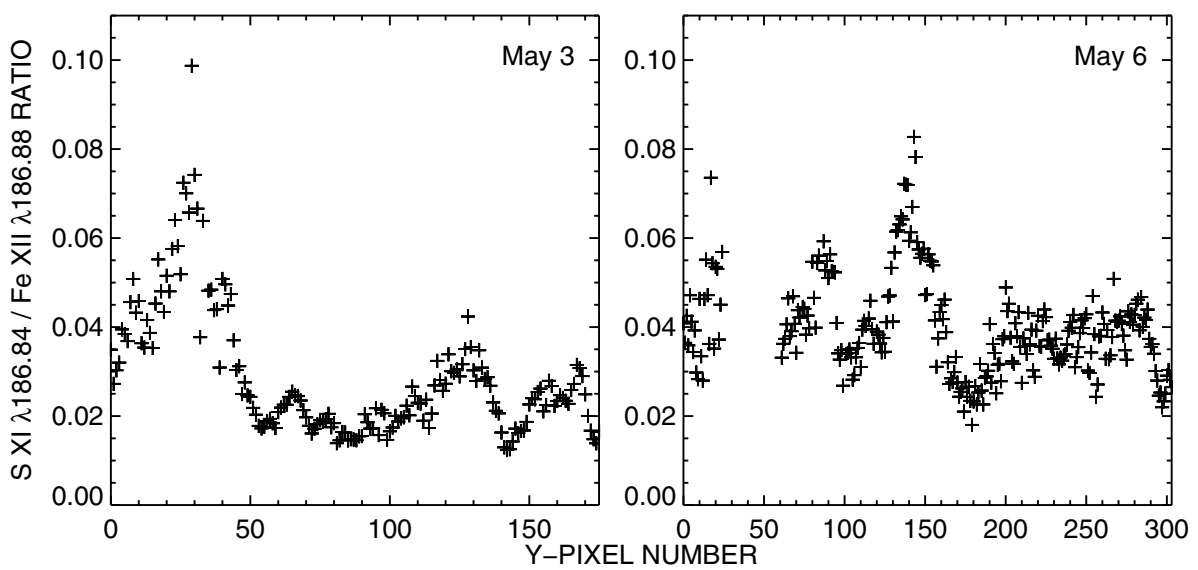

Fig. 5. The intensity ratio of S XI $\lambda 188.64$ to the blended FeXI $\lambda 186.88$ feature for two columns of spatial pixels in the May 3 (left panel) and May 6 (right panel) data sets. The $\lambda 188.64$ intensity has been estimated from fits to S XI $\lambda 188.68$. For the May 6 data set, accurate fits to $\lambda 188.68$ were not possible due to a dust particle on the CCD at this position.

\subsection{FeXII 195.12}

The Fe XII emission line at $195.12 \AA$ is usually the strongest line observed by EIS and it dominates the spectrum in the region 194-196 A. Despite this, some care has to be taken when automatically fitting the line. Firstly, there is another Fe XII transition at $195.18 \AA$ which lies within the $\lambda 195.12$ profile and thus distorts the observed line profile from a Gaussian shape. This line is predicted by CHIANTI to be $\leq 10 \%$ of the strength of $\lambda 195.12$ below $10^{10} \mathrm{~cm}^{-3}$, but this increases to $22 \%$ at $10^{11} \mathrm{~cm}^{-3}$ and so can not be ignored if the data set shows high densities. A detailed discussion of the effects of the blending $\lambda 195.18$ line is provided in Appendix A.

In addition to the Fe XII blending component there are a number of weak lines either side of $\lambda 195.12$ in the spectrum, as can be seen in the two panels in Fig. 6. Simply performing a single Gaussian fit over the wavelength range 194.80-195.50 A, for example, will lead to an incorrrect fit to the spectrum background which will result in the intensity of the $\lambda 195.12$ line being underestimated at the $1-3 \%$ level.

The solution to these two problems in the present work is as follows. Spectral regions are identified that are free from emission lines and are used to constrain the continuum level. The regions containing the weak lines are not included in the fit. This is illustrated in Fig. 6 where crosses indicate the pixels that have been used for the Gaussian fitting. To deal with the $\lambda 195.18$ blend a two Gaussian fit has been performed. The stronger $\lambda 195.12$ line has free parameters for the centroid, width and amplitude, while $\lambda 195.18$ is forced to be $0.06 \AA$ to the long wavelength side of $\lambda 195.12$ (the CHIANTI wavelengths for the two transitions are 195.119 and $195.179 \AA$ ), and to have the same width as $\lambda 195.12$. The amplitude of $\lambda 195.18$ is free to vary.

While $\lambda 195.12$ is the strongest Fe XII line observed by EIS and thus likely to be the most commonly used line for density diagnostics, there are two other strong Fe XII lines at $192.39 \AA$ and $193.51 \AA$ that are density insensitive relative to $\lambda 195.12$ and thus can be substituted for this line to be used as density diagnostics relative to $\lambda 186.88$ and $\lambda 196.64$. These lines are unblended and are less likely to saturate on the detector than $\lambda 195.12$ since they are both weaker transitions and the EIS effective area is lower at these wavelengths. $\lambda 192.39$ and $\lambda 193.51$ are discussed in Appendix B where it is shown that the ratios of $\lambda 192.39$, $\lambda 193.51$ and $\lambda 195.12$ closely match the predictions of CHIANTI and thus they all can be used in density diagnostics.

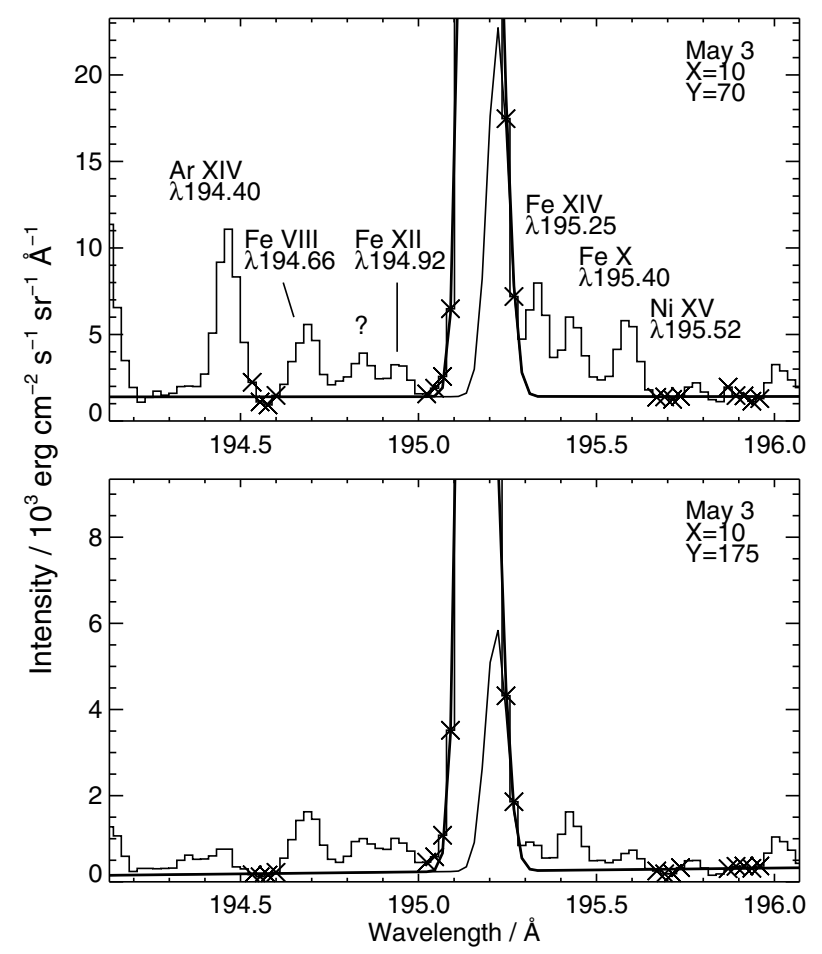

Fig. 6. Two example spectra from the May 3 data set showing the emission lines neighbouring the strong Fe XII $\lambda 195.12$ line. A two Gaussian fit has been performed to $\lambda 195.12$ and the neighbouring Fe XII $\lambda 195.18$ line. The crosses show the wavelength pixels that have been included in the fit, and the thick line shows the fit result. A thin line is used to highlight the weaker Gaussian that represents $\lambda 195.18$. Note that there are a number of weak lines neighbouring the Fe XII feature that potentially affect the fits. The peak of the $\lambda 195.12$ line extends by a factor five beyond the top of each of the two plots.

\subsection{FeXIII 196.54, FeXII 196.64}

These two lines are fitted simultaneously with a double Gaussian, with each parameter free to vary. The main difficulty is to identify a clean continuum area in the spectrum for accurately fitting the background for the fits. To the long wavelength side of $\lambda 196.64$ there are three lines: S X $\lambda 196.81$, Fe XII $\lambda 196.92$ and an unknown line at around 197.02 $\AA$ (Brown et al. 2008). Beyond this latter line there is a small region at around 197.2 $\AA$ which is free of lines and suitable for estimating the background level. On the short wavelength side of $\lambda 196.54$ there is a Fe VII line at $196.42 \AA$ (Brown et al. 2008) but this is generally negligible in active region conditions. 


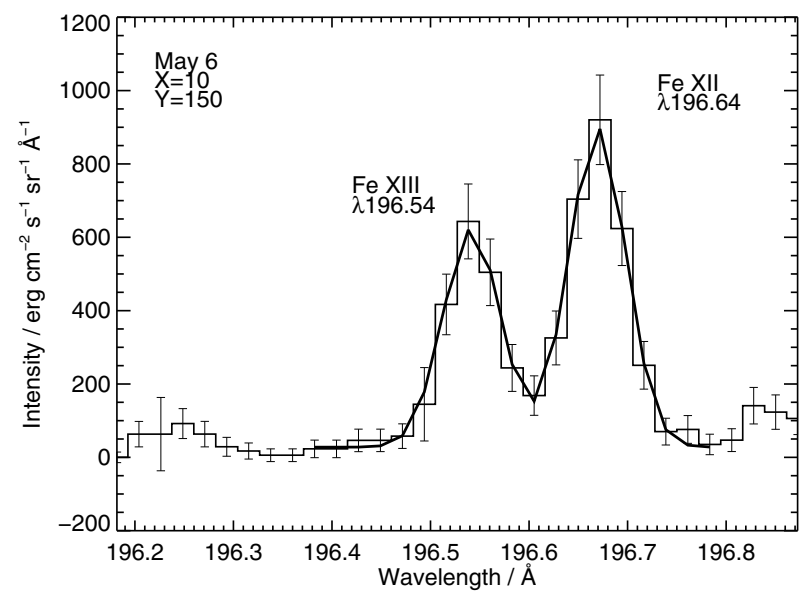

Fig.7. An example double-Gaussian fit to FeXIII $\lambda 196.54$ and Fe XII $\lambda 196.64$ from the May 6 data set. The chosen spatial pixel is indicated. The data are plotted as a thin line and the fit with a thick line. The error bars on the intensity measurements are also shown.

For the fits performed in this work, only the pixels directly neighbouring the Fe XII and Fe XIII lines were used for estimating the background level (Fig. 7) as the wavelength window was not wide enough to observe the background region around 197.2 A. A uniform background was assumed for the fitting rather than a linear fit in order to prevent the S X $\lambda 196.81$ line distorting the derived background.

In regions where there is an absence of hot plasma and the density is low, the Fe XIII $\lambda 196.54$ line can be very weak and thus difficult to fit, but this was a not a problem for the data sets considered here.

Brown et al. (2008) list a Fe VIII line at $196.65 \AA$ which thus blends with Fe XII $\lambda 196.64$. CHIANTI predicts the Fe VIII line to be a factor 0.06 weaker than Fe VIII $\lambda 194.66$ (visible in Fig. 6), and thus it would be expected to be negligible in most circumstances. However, in active regions Fe VIII is often seen to be strongly enhanced in small brightenings or loop footpoints (Young et al. 2007a), and in these regions $\lambda 196.65$ can be significant. Examples are found in both the May 3 and May 6 data sets where methods of correcting the measured $\lambda 196.64$ intensity for the Fe VIII contribution are discussed.

\subsection{FeXIII 202.04}

Fe XIII $\lambda 202.04$ is unblended and can be fit with a single Gaussian. We choose to fit a single Gaussian to the wavelength region 201.90-202.32 Å (Fig. 8).

\subsection{FeXIII 203.82}

This feature is a self-blend of two Fe XIII lines with CHIANTI wavelengths of 203.797 and $203.828 \AA$. The two lines have weak density dependence (Fig. 10), with the ratio almost constant above $10^{9.5} \mathrm{~cm}^{-3}$. There is an additional line due to Fe XII found at $203.728 \AA$ which is not clearly separated from the Fe XIII lines (Fig. 9). Generally this line is always weaker than the Fe XIII feature, but in quiet Sun conditions it can be comparable in strength.

The fitting method chosen for these lines is to fit the wavelength region 203.5-204.1 $\AA$ with three Gaussians each set to the same width (which is free to vary). The two Fe XIII components are assumed to have a fixed ratio $\lambda 203.797 / \lambda 203.828$ of 0.40 . Although Fig. 10 demonstrates that this is only valid

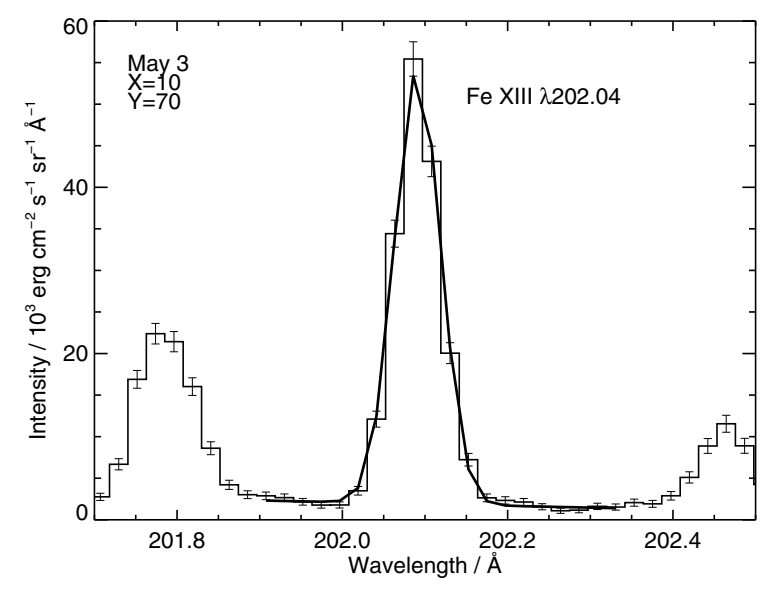

Fig. 8. An example single Gaussian fit to the Fe XIII $\lambda 202.0$ emission line. The May 3 data set is used, and the image pixel indicated.

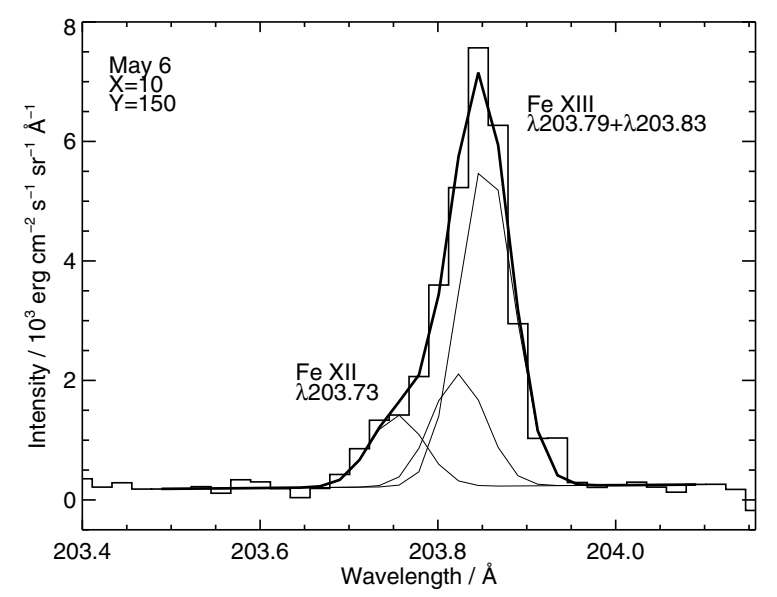

Fig. 9. An example fit to the feature at $203.8 \AA$ that comprises Fe XII $\lambda 203.72$, and Fe XIII $\lambda \lambda 203.79,203.83$ from the May 6 data set. The spatial pixel chosen is indicated. Three thin lines are used to show the three individual Gaussians from the fit.

for $\log N_{\mathrm{e}} \gtrsim 9.0$, the assumption is preferable to allowing the line ratio to freely vary when performing automatic fits to the data. The close separation of the two Fe XIII lines also means that their precise ratio is not crucial to fitting the spectral feature. The peak of the Fe XII component is free to vary in the fit, but the centroid is fixed relative to the $203.828 \AA$ line using the separation from the CHIANTI database. Fe XIII $\lambda 203.797$ also has a fixed centroid relative to the $\lambda 203.828$ line. In all there are four free parameters, with two additional parameters for a linear fit to the background.

\section{Quiescent active region data set (2007 May 6)}

The data sets presented in this and the next section are from active region NOAA AR 10963 which rotated across the solar disc during 2007 April 24 to May 9. On May 6 the region was close to the west limb and observed by EIS with the study AR_VELOCITY_MAP which consisted of an initial context raster with the $40^{\prime \prime}$ slit, followed by a long duration $1^{\prime \prime}$ slit raster covering a region $330^{\prime \prime} \times 304^{\prime \prime}$. Figure 11 shows an image from the $1^{\prime \prime}$ raster formed from Fe XII $\lambda 195.12$. The vertical black bands arise from Hinode passing behind the Earth as the raster progressed (the observation was taken during the Hinode eclipse season). Gaussian fits were performed on the spatial region highlighted in Fig. 11. The region has size $21^{\prime \prime} \times 304^{\prime \prime}$ and 


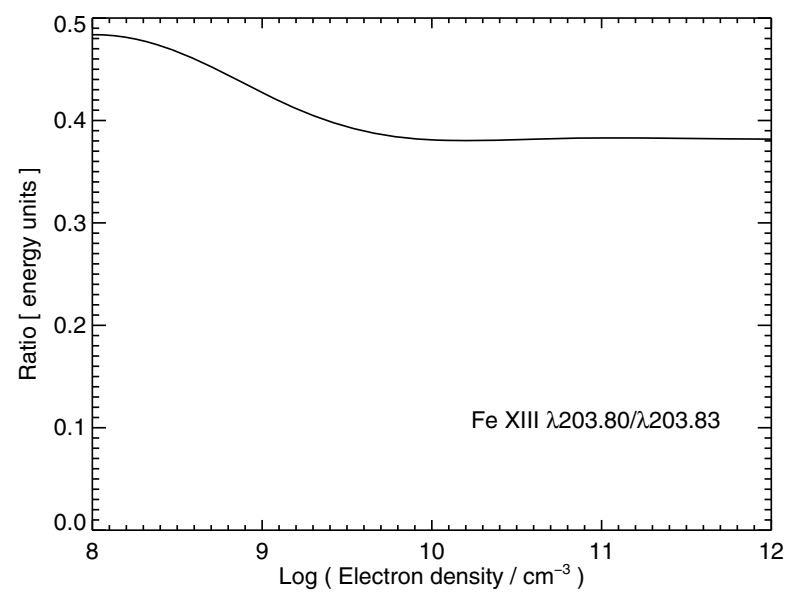

Fig. 10. Theoretical variation of the Fe XIII $\lambda 203.80 / \lambda 203.83$ ratio derived from CHIANTI.

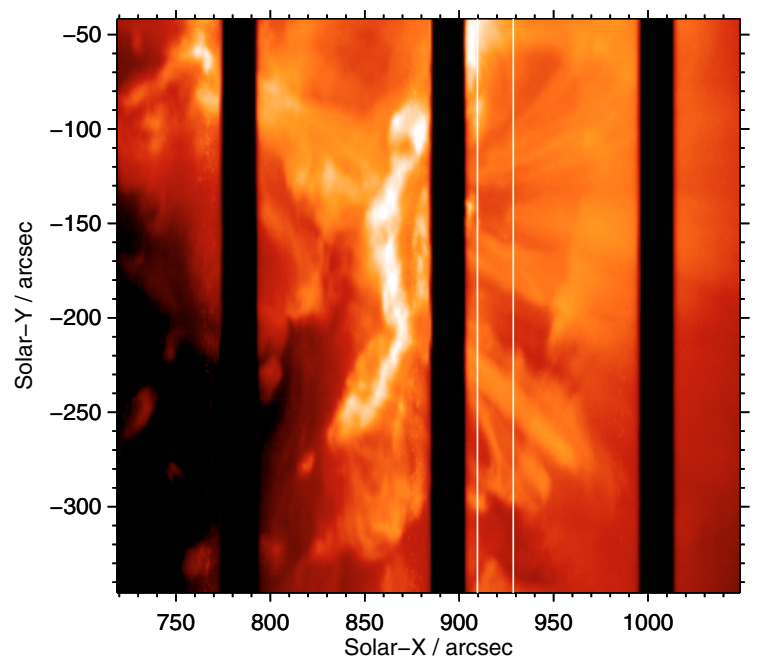

Fig. 11. Image of AR 10963 obtained in the Fe XII $\lambda 195.12$ line on May 6. The raster began at 18:19 UT and completed at 23:13 and was obtained during the Hinode eclipse season. The three thick, black, vertical lines show when the satellite passed behind the Earth. The two thin vertical black lines indicate the spatial region used for the analysis in Sect. 6.

it crosses a filament and some coronal loops. At each pixel in this sub-region each of the density diagnostic lines discussed in Sect. 5 has been fitted. We consider a slice through the data set at $X$-pixel 10 and present the derived densities from the Fe XII and Fe XIII lines in Figs. 12 and 13, respectively. Densities calculated with the Keenan et al. (2007) Fe XIII atomic model are shown in Fig. 15 (available online). The upper panel in each plot shows the images derived from the Fe XII $\lambda 195.12$ and Fe XIII $\lambda 202.04$ lines, respectively, with the selected image column indicated.

Considering first the Fe XII ratios $\lambda 186.88 / \lambda 195.12$ and $\lambda 196.64 / \lambda 195.12$, the line intensities from the fitting methods described in the previous sections are used for each line. $\lambda 186.88$ is not corrected for the SXI $\lambda 186.84$ contribution (Sect. 5.1), but $\lambda 196.64$ is corrected for the Fe VIII $\lambda 196.65$ contribution (Sect. 5.3). The correction was performed by fitting the Fe VIII $\lambda 194.66$ emission line at each pixel in the data set. The fitting method for this line was similar to the nearby Fe XII $\lambda 195.12$ (Sect. 5.2), with only pixel sections chosen for the line and the background regions at 194.5, 195.7 and $195.9 \AA$. From CHIANTI, the Fe VIII $\lambda 196.65 / \lambda 194.66$ ratio is insensitive to density and has a theoretical value of 0.058 . Thus the Fe XII $\lambda 196.64$ intensity was derived by subtracting 0.058 times the intensity of Fe VIII $\lambda 194.66$ at each pixel. The maximum contribution of Fe VIII to the measured line intensity of the $196.64 \AA$ feature is found to be $9 \%$ using this method.

The measured intensity ratios were converted to densities using the CHIANTI database, and are plotted in Figs. 12 and 13. The $1 \sigma$ error bars on the intensities have been translated to errors on the derived densities, and thus vertical lines are used on these plots, connecting the lower limit to the upper limit. The densities track each other reasonably well along the slit, with the positions of peaks and troughs in good agreement. However, the $\lambda 196.64 / \lambda 195.12$ ratio generally gives higher densities than $\lambda 186.88 / \lambda 195.12$, and particularly so at certain of the peaks along the slit, e.g., around pixels 170, 200 and 240.

The dashed line in Fig. 12 shows the variation of Fe VIII $\lambda 194.66$ along the slit, and it is clear that the largest discrepancies between the two Fe XII ratios occur where Fe VIII $\lambda 194.66$ is strongest, implying that the Fe VIII contribution to Fe XII $\lambda 196.64$ is not fully accounted for. This suggests that there are problems with the Fe VIII atomic model in CHIANTI. It is to be noted that adjusting the theoretical Fe VIII $\lambda 196.65 / \lambda 194.66$ ratio to around 0.25 brings the $\lambda 196.64 / \lambda 195.12$ and $\lambda 186.88 / \lambda 195.12$ densities into good agreement.

The two Fe XIII density sensitive ratios show excellent agreement (Fig. 13), except in the pixel region 0 to 30 where the $\lambda 203.82 / \lambda 202.04$ ratio is lower by $0.2-0.3 \mathrm{dex}$, although the errors bars on the $\lambda 196.54 / \lambda 202.04$ ratio are significantly larger in this region. At all pixels the $\lambda 196.54 / \lambda 202.04$ ratio yields a marginally higher density than $\lambda 203.82 / \lambda 202.04$. Densities derived from the Keenan et al. (2007) atomic model (Fig. 15) are very similar, being around 0.1 dex lower than for the CHIANTI model, and the two ratios are in even better agreement, yielding almost identical densities along the EIS slit.

In Fig. 14 we compare the densities from Fe XII $\lambda 186.88 / \lambda 195.12$ and Fe XIII $\lambda 203.82 / \lambda 202.04$. (We choose these two ratios for the comparison due to the Fe VIII correction problem for $\lambda 196.64 / \lambda 195.12$ discussed earlier, and the fact that Fe XIII $\lambda 203.82$ is a stronger line than $\lambda 196.54$ at low densities and thus yields densities with smaller error bars.) The Fe XII ratio yields higher densities at all pixels by up to 0.4 dex, and we also note that Fe XIII generally shows less variation pixel-to-pixel than Fe XII. These differences will be discussed further in Sect. 9.

\section{High-density data set (2007 May 3)}

The May 6 data set demonstrates the use of the Fe XII and Fe XIII diagnostics in a quiescent part of an active region, typical of large, developed coronal loops. The two sets of ion ratios are sensitive to higher densities, and so the EIS data archive was searched for examples of high densities. On 2007 May 3, the same active region showed small flaring activity and this was captured with the EIS study DIAG_40X180_S0_30S which consists of two rasters, the first being the same 40" slot context raster used by the AR_VELOCITY_MAP study, and the second being a raster covering an area $40^{\prime \prime} \times 176^{\prime \prime}$ using the $1^{\prime \prime}$ slit, the latter having an identical line list to the narrow slit raster used on May 6.DIAG_40X180_S0_30S was run during the period 00:07 to $05: 28$ UT with the slot context raster run once at the start of the period, and the narrow slit raster repeated 15 times. A GOES class C9 flare began at May 2 23:15 UT, peaked at 23:50 UT and decayed by May 3 02:00 UT. The first narrow slit raster 

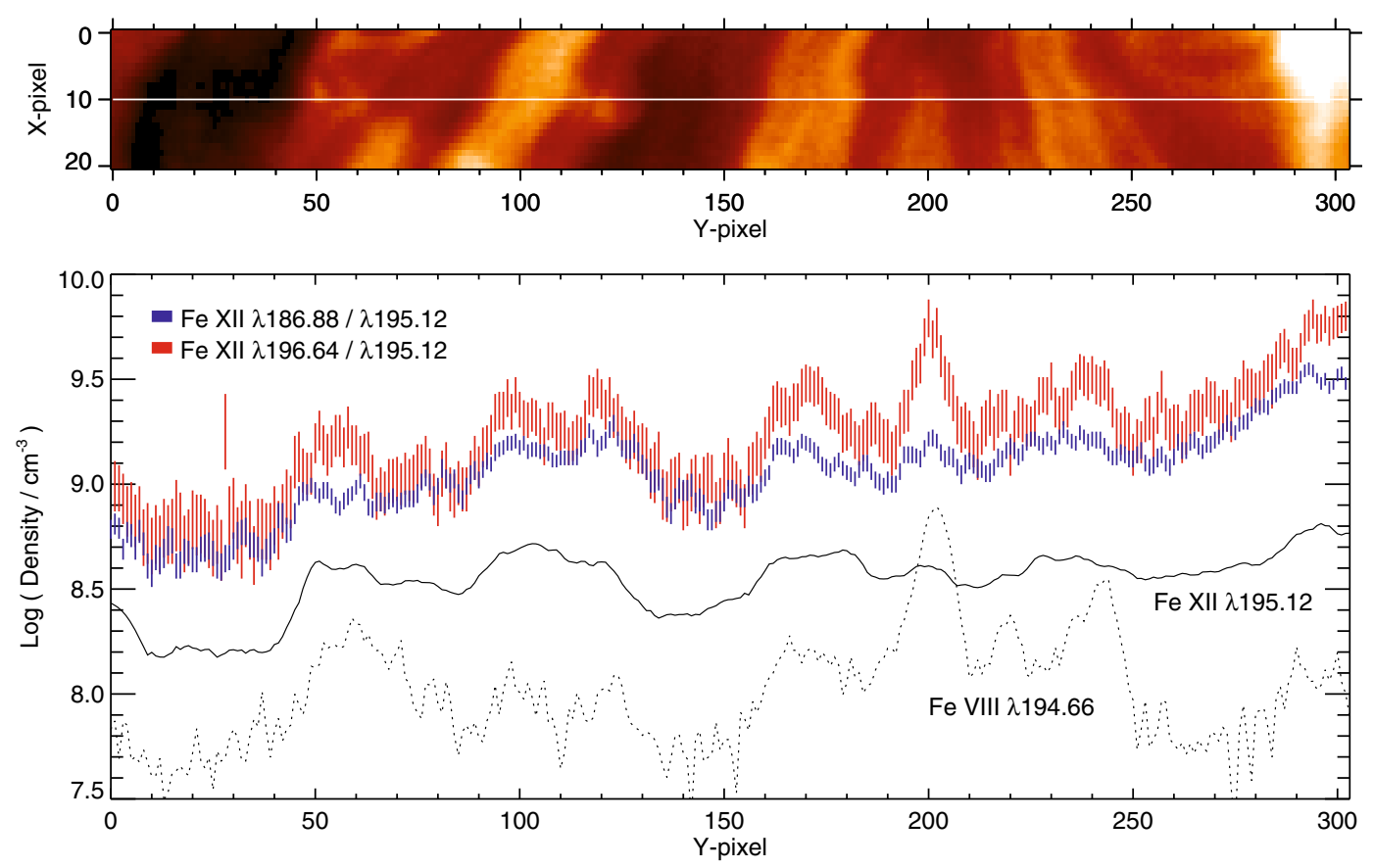

Fig. 12. The upper panel shows an image from the May 6 data set obtained in the Fe XII $\lambda 195.12$ line. The horizontal line indicates the data column chosen to derive densities. The lower panel shows densities derived from the Fe XII $\lambda 186.88 / \lambda 195.12$ (blue) and $\lambda 196.64 / \lambda 195.12$ (red) density diagnostics. Short vertical lines are used indicating the lower and upper densities based on the $1 \sigma$ error bars on the measured line ratios. The black line shows the variation of the $\lambda 195.12$ line intensity, while the dashed line shows the variation of the Fe VIII $\lambda 194.66$ line. For the latter two lines the quantity plotted is $\log \left(10^{5} \mathrm{I}\right)$ and $\log \left(10^{5.5} \mathrm{I}\right)$, respectively, where $I$ is the line intensity in units $\mathrm{erg} \mathrm{cm}^{-2} \mathrm{~s}^{-1} \mathrm{sr}^{-1}$.
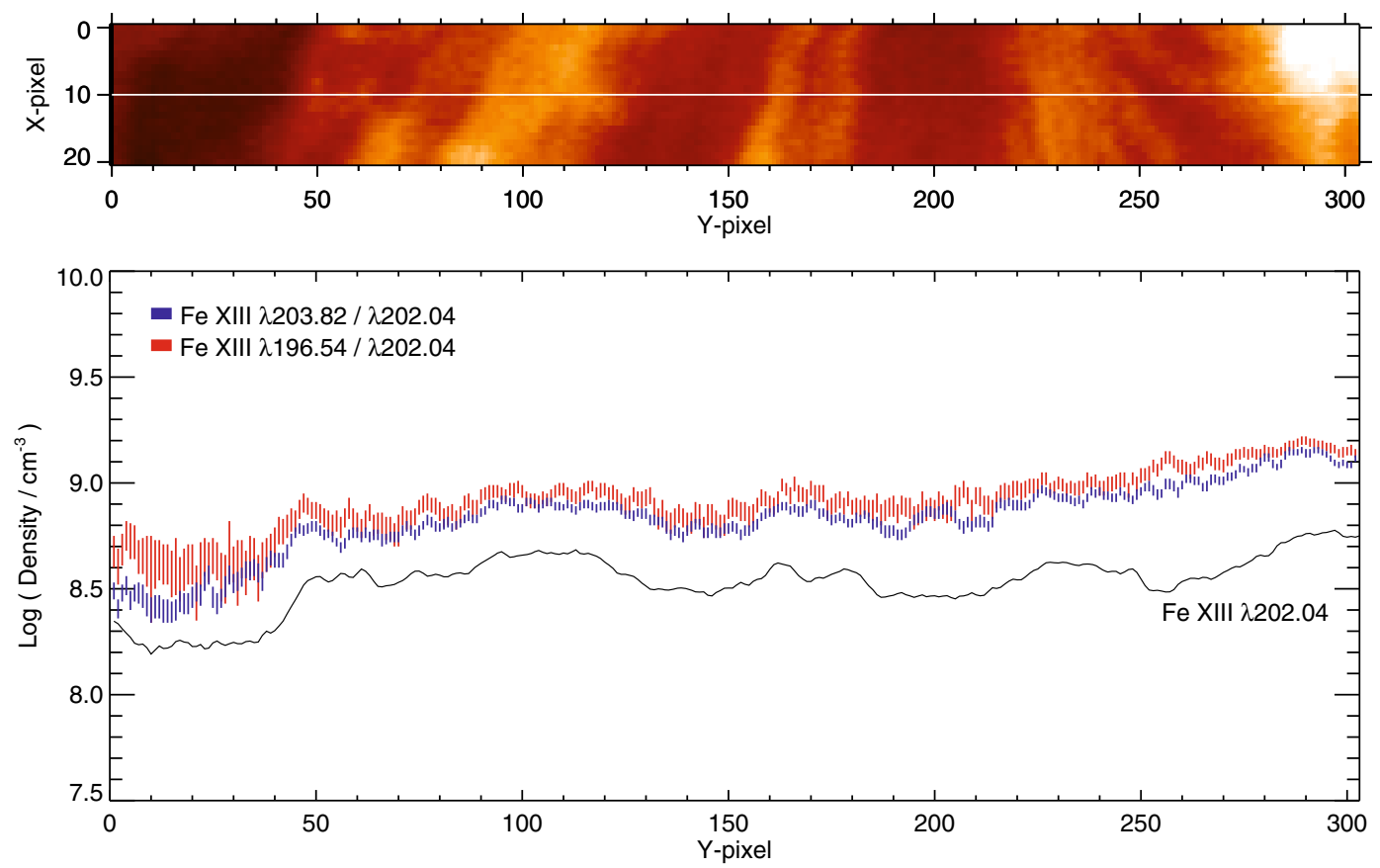

Fig. 13. The same plot as Fig. 12 except for Fe XIII. In the upper panel the image shown is from Fe XIII $\lambda 202.02$. In the lower panel the red lines show densities derived from the $\lambda 196.54 / \lambda 202.02$ ratio and the blue lines shows densities from the $\lambda 203.82 / \lambda 202.02$ ratio. The black line shows the variation in intensity of Fe XIII $\lambda 202.04$, where the plotted quantity is $\log \left(10^{5} I\right)$.

ran during the period 00:11 UT to 00:32 UT during the decay phase of the flare and the flare line Ca XVII $\lambda 192.82$ is prominent in the spectrum. Figure 16 shows an image obtained with the context slot raster at 00:07 UT and reveals the large-scale structure of the active region. The image is from Si X $\lambda 261.06$ as the Fe XII and Fe XIII lines are saturated due to the flare in this data set. Saturation of $\lambda 195.12$ also occurs in the brightest parts of the narrow slit raster image, and so for our density study a data column is selected that is to one side of the very brightest regions in the image (indicated in the upper panels of Figs. 17 and 18). Even in this data column, one or two pixels in the centre of the $\lambda 195.12$ profile are saturated at the brightest part of the image, over $Y$-pixels 54 to 69 . Surprisingly, though, the two Gaussian fit to the $195 \AA$ feature still yielded good results. This was checked 


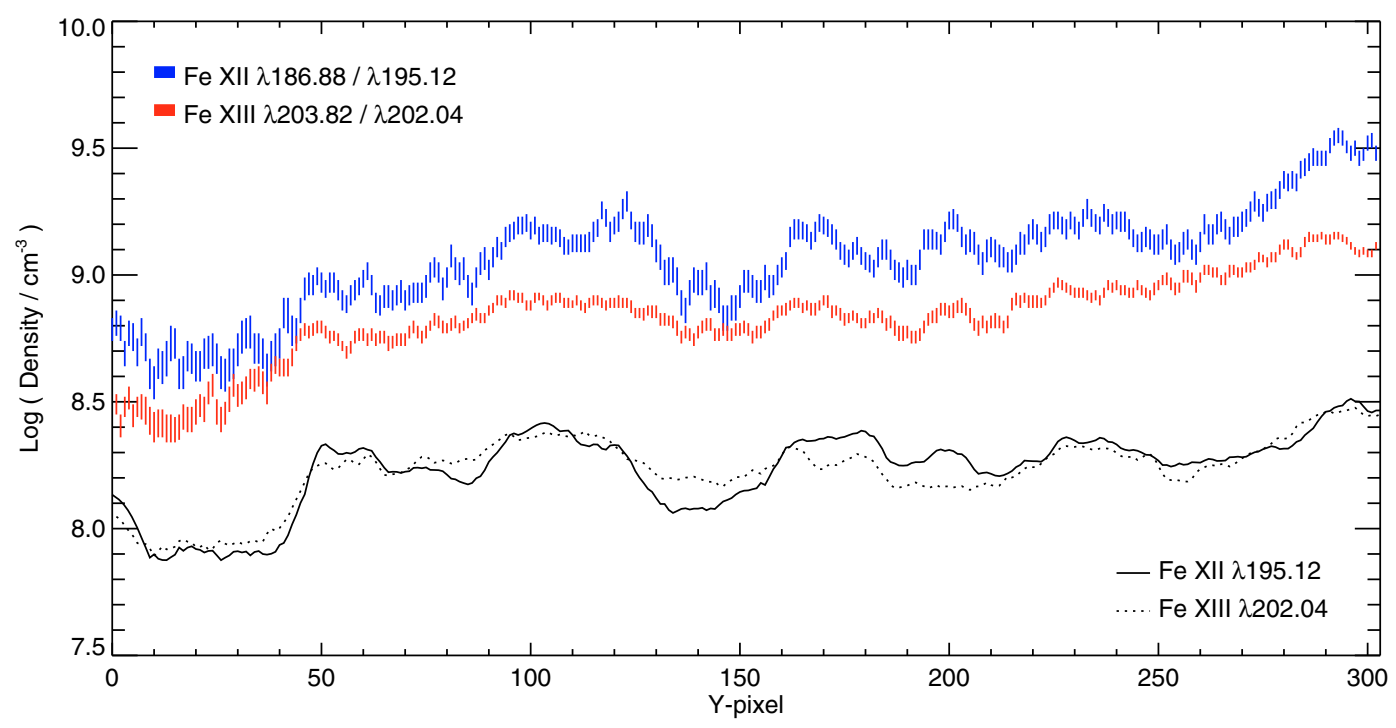

Fig. 14. A comparison of densities derived from the Fe XII $\lambda 186.88 / \lambda 195.12$ (blue lines) and Fe XIII $\lambda 203.82 / \lambda 202.04$ (red lines) ratios. The black solid line shows the variation of the Fe XII $\lambda 195.12$ intensity, while the black dashed line shows the variation of the Fe XIII $\lambda 202.04$ intensity. For the latter two lines the quantity plotted is $\log \left(10^{5.2} \mathrm{I}\right)$, where $I$ is the line intensity in units erg $\mathrm{cm}^{-2} \mathrm{~s}^{-1} \mathrm{sr}^{-1}$.
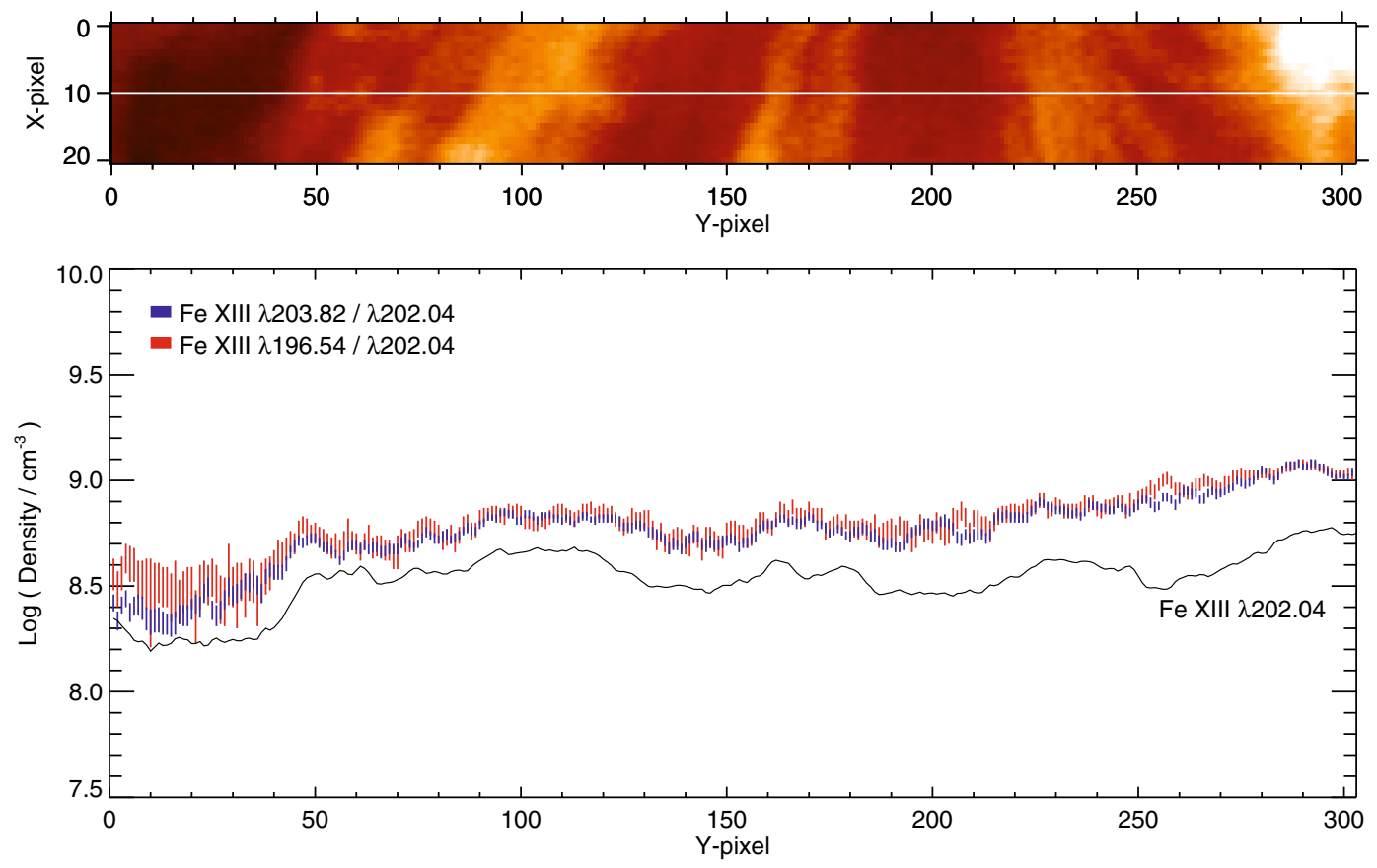

Fig. 15. Exactly analogous plot to Fig. 13, except the densities are derived using the Keenan et al. (2007) Fe XIII atomic model.

in two ways. Firstly the insensitive $\lambda 192.39 / \lambda 195.12$ ratio was plotted along the data column (Appendix B) and no significant feature is found over the saturated region. Secondly, the density was re-computed using the $\lambda 186.88 / \lambda 192.39$ ratio, and differences of at most 0.15 dex were found.

As for the May 6 data set, Fe XII $\lambda 196.64$ has been corrected for the Fe VIII blend using the $\lambda 194.66$ line, although the Fe VIII contribution to the $\lambda 196.64$ feature is at most $5 \%$ here.

$X$-pixel 10 in the May 3 data-set was selected for the density analysis, and the densities from the pairs of Fe XII and Fe XIII ratios are presented in Figs. 17 and 18. The Fe XIII densities derived from the Keenan et al. (2007) atomic model are presented in Fig. 20 (available online).

The derived Fe XII densities again track each other reasonably well, with $\lambda 196.64 / \lambda 195.12$ yielding higher densities by up to 0.4 dex, with the largest discrepancies being at high densities (Fig. 17). A strong peak in the Fe VIII $\lambda 194.66$ line is seen around $Y$-pixel 57 which may partly account for the density discrepancy here, but at other locations this can not be the case.

For Fe XIII the two ratios again track each other very well at low densities (Fig. 18), but at high densities $\lambda 203.83 / \lambda 202.04$ is found to get close to the high-density limit of $\log N_{\mathrm{e}}=11.3$ of the ratio (indicated by the large error bars). This does not happen for $\lambda 196.54 / \lambda 202.04$ which has greater sensitivity to high densities. This issue will be discussed further in Sect. 9. The same problem occurs when using the Keenan et al. (2007) atomic model (Fig. 20), although as the $\lambda 203.82 / \lambda 202.04$ high-density limit is slightly larger for this model (Fig. 1) then the discrepancy between the two ratios is not as large at high densities. Densities from $\lambda 196.54 / \lambda 202.04$ are 0.2 dex lower at the highest densities 


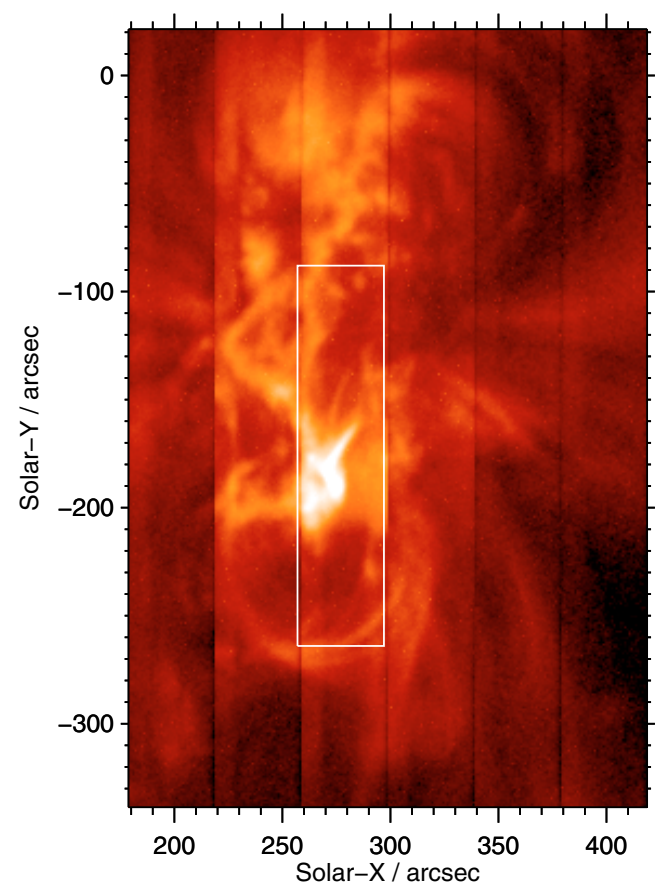

Fig. 16. Context image of AR 10963 obtained on 2007 May 3 00:03 UT, shortly before the raster analysed in Sect. 7 . The image was obtained by making a six exposure raster with the $40^{\prime \prime}$ slit. The image is formed in the emission line Si X $\lambda 261.06$ which is formed at a similar temperature to Fe XII $\lambda 195.12$. The box indicates the location of the narrow slit raster that followed the context raster

using the Keenan et al. (2007) model compared to the CHIANTI model.

In Fig. 19 the densities from Fe XII $\lambda 186.88 / \lambda 195.12$ and Fe XIII $\lambda 196.54 / \lambda 202.04$ are compared. $\lambda 196.54 / \lambda 202.04$ is preferred to $\lambda 203.82 / \lambda 202.04$ for Fe XIII as we believe $\lambda 203.82 / \lambda 202.04$ is not accurately measuring the density for $\log N_{\mathrm{e}} \geq 10.0$ (Sect. 9). The discrepancies noted for the May 6 data set are also seen here, with Fe XII yielding higher densities by up to 0.5 dex. If the Keenan et al. (2007) model is used the difference between Fe XII and Fe XIII rises to 0.7 dex. The magnitude of this difference will be discussed in Sect. 9.

\section{Integrity of the line fit parameters}

The density analysis presented in the previous sections used the emission line intensities that are derived from Gaussian fits to the emission lines. The fits also yield centroid and line width information for each line and these are valuable for assessing the quality of the line fits and for revealing any unaccounted for line blending. Each of the three Fe XII lines and each of the three Fe XIII lines would be expected to show the same line width and velocity behaviour across the data and the plots in Fig. 21 show comparisons for the various line pairs. For Fe XII, the comparisons are done against $\lambda 195.12$ (the eight panels on the left side of Fig. 21), while for Fe XIII they are done against $\lambda 202.04$ (the eight panels on the right side of Fig. 21). It is to be noted that the measured $\lambda 186.88$ line is a blend of two Fe XII emission lines separated by $0.033 \AA$ which thus affects the width and velocity measurements. Fe XIII $\lambda 203.82$ was assumed to be a blend of two Fe XIII lines when calculating densities, however, in the Gaussian fitting (Sect. 5.5) the line properties of both components were derived thus the comparisons in Fig. 21 are for the longer wavelength $\lambda 203.828$ component.
Considering first the line widths (the full width at half maximum, FWHM, in this case) we plot the widths of the line of interest against the reference line in row 1 and row 3 of Fig. 21 for the May 6 and May 3 data sets, respectively. Ideally the points should lie on a diagonal line running from the bottom-left to top-right, indicating that all the Fe XII or Fe XIII lines broaden together. For the May 6 data set the line width of $\lambda 195.12$ is very consistent along the slit, with an average width of $66.0 \pm$ $1.3 \mathrm{~m} \AA$. However, both $\lambda 186.88$ and $\lambda 196.64$ show a significantly broader spread, with average values of $78.0 \pm 4.1 \mathrm{~m} \AA$ and $67.3 \pm 4.5 \mathrm{~m} \AA$. The greater spread is simply due to the lower signal-to-noise of the two lines. The $\lambda 196.64$ and $\lambda 195.12$ widths are in excellent agreement, while the larger width of $\lambda 186.88$ arises from the fact that it is a blend of two lines with wavelengths 186.85 and $186.89 \AA$.

For Fe XIII, the greater spread of widths for $\lambda 203.83$ and $\lambda 196.54$ are again explained by the lower signal-to-noise in these lines. The average width for $\lambda 202.04$ is $67.4 \pm 1.7 \mathrm{~m} \AA$, while for $\lambda 203.83$ and $\lambda 196.54$ they are $69.9 \pm 3.4 \mathrm{~m} \AA$ and $63.2 \pm 5.0 \mathrm{~m} \AA$, respectively. The latter is noticeably lower than the other width measurements, although it is consistent within the error bars.

Moving to the May 3 data set, a greater spread of line widths is found particularly for Fe XII. The average widths for $\lambda 195.12$, $\lambda 186.88$ and $\lambda 196.64$ are $67.9 \pm 3.1,84.0 \pm 4.4$ and $69.4 \pm 4.2 \mathrm{~m} \AA$, respectively. The average $\lambda 186.88$ width is significantly larger than for May 6 due to an increasing contribution of the $186.85 \AA$ component at high densities. The other two lines have comparable widths to May 6, only slightly broader as might be expected for this flare data set. The average widths for Fe XIII $\lambda 202.04$, $\lambda 196.54$ and $\lambda 203.83$ are $67.5 \pm 2.8,66.0 \pm 3.9$ and $71.0 \pm 3.1 \mathrm{~mA}$, respectively. We note that, as with the May 6 data set, $\lambda 203.82$ is a little broader than the other lines which implies the three Gaussian model for the lines at this wavelength may not be correct. Either an unaccounted for blending line, or errors in one or more of the rest wavelengths could be responsible. The effect, however, is small.

For comparing the line centroid measurements we consider the line velocities as given by

$v=c \frac{\lambda-\lambda_{\text {ref }}}{\lambda_{\text {ref }}}$

where $c$ is the speed of light, $\lambda$ is the measured emission line centroid and $\lambda_{\text {ref }}$ is the reference wavelength of the line (taken from CHIANTI). The positions of the EIS emission lines on the detector vary over the orbit by $\approx \pm 1$ pixels (Brown et al. 2007), and so the measured centroids are normalised here relative to the reference lines ( $\lambda 195.12$ for Fe XII and $\lambda 202.04$ for Fe XIII), i.e., the wavelengths of these lines along the EIS slit are assumed to average to the reference wavelengths of the lines. As for the line widths, the line velocities should ideally fall on a diagonal line running from bottom-left to top-right in the figures, indicating that each line should reveal the same blueshift or redshift. In addition the diagonal line should pass through $(0,0)$ if the reference wavelengths of the lines are both correct.

For the May 6 data set there is little dynamic activity along the slit which is revealed in the $\lambda 202.04$ and $\lambda 195.12$ measurements which each have a very narrow spread of values. The other lines show a broader spread of values due to the lower signalto-noise of these lines. Fe XII $\lambda 196.64$ shows a small clump of values around $+20 \mathrm{~km} \mathrm{~s}^{-1}$ which is caused by the blending Fe VIII line mentioned in Sect. 5.3. Of the four comparison plots for May 6, only for $\lambda 203.83$ are the points close to 

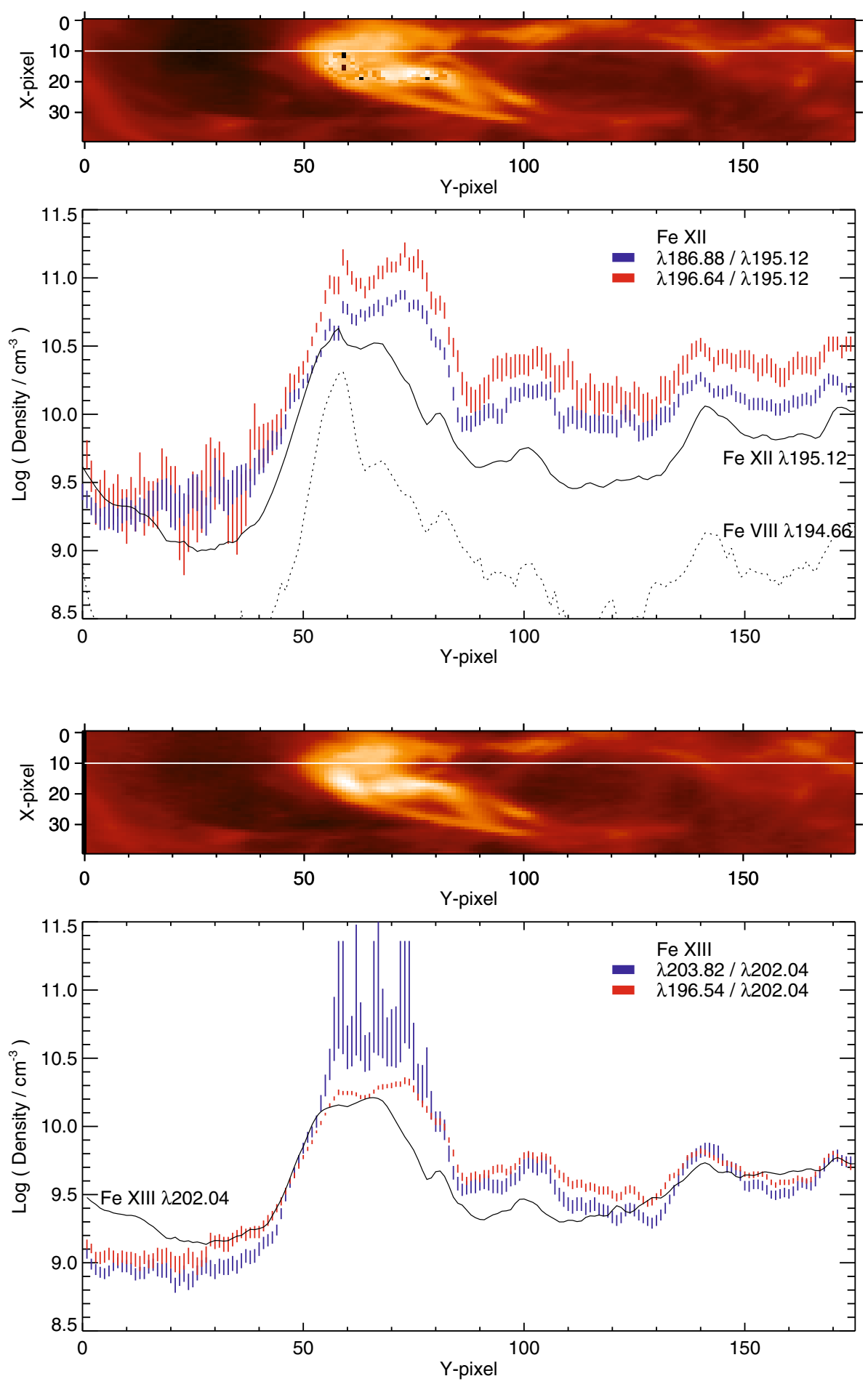

Fig. 17. The same plot as Fig. 12 except this time for the May 3 data set. For the intensity curves from FeXII $\lambda 195.12$ and Fe VIII $\lambda 194.66$, the quantities plotted are $\log \left(10^{6.5} I\right)$ and $\log \left(10^{7} I\right)$, respectively, where $I$ is the line intensity in units erg $\mathrm{cm}^{-2} \mathrm{~s}^{-1} \mathrm{sr}^{-1}$.

Fig. 18. The same plot as Fig. 13 except this time for the May 3 data set. For the intensity curve from Fe XIII $\lambda 202.04$ the quantity plotted is $\log \left(10^{6.5} I\right)$ where $I$ is the line intensity in units erg $\mathrm{cm}^{-2} \mathrm{~s}^{-1} \mathrm{sr}^{-1}$.

the $(0,0)$ point, indicating accurate reference wavelengths. For $\lambda 186.88$ the $\approx-20 \mathrm{~km} \mathrm{~s}^{-1}$ offset is explained by the blending $186.85 \AA$ line. For $\lambda 196.64$ the average offset is $10.9 \pm 3.4 \mathrm{~km} \mathrm{~s}^{-1}$ which suggests a revised reference wavelength for the line of 196.647 $\AA$ (assuming the CHIANTI wavelength for $\lambda 195.12$ is correct). This compares with the value of $196.645 \AA$ in the quiet Sun spectrum of Brown et al. (2008). The average offset for Fe XIII $\lambda 196.54$ is $-33.9 \pm 3.9 \mathrm{~km} \mathrm{~s}^{-1}$, suggesting a revised reference wavelength of $196.518 \AA$ (assuming the CHIANTI wavelength for $\lambda 202.04$ is correct). The average value in the five spectra presented by Brown et al. (2008) is $196.520 \AA$. The uncertainties in the reference wavelengths of Fe XII $\lambda 195.12$ and
Fe XIII $\lambda 202.04$ are $\pm 0.002 \AA$ (Del Zanna \& Mason 2005; Brown et al. 2007), thus our revised wavelengths for Fe XII $\lambda 196.64$ and Fe XIII $\lambda 196.54$ are $196.647 \pm 0.003 \AA$ and $196.518 \pm 0.003 \AA$, respectively.

The line velocity plots from the May 3 data set in the bottom row of Fig. 21 are quite striking. There is clearly significant dynamic activity in the data set which is actually related to the high density regions around $Y$-pixels 40 to 90 . Reassuringly a clear bottom-left to top-right diagonal is seen in each plot, demonstrating that the Fe XII and Fe XIII lines are responding to the velocity shifts in unison. The effect is particularly clear for Fe XIII $\lambda 203.83$ and $\lambda 202.04$. In addition to the diagonal 

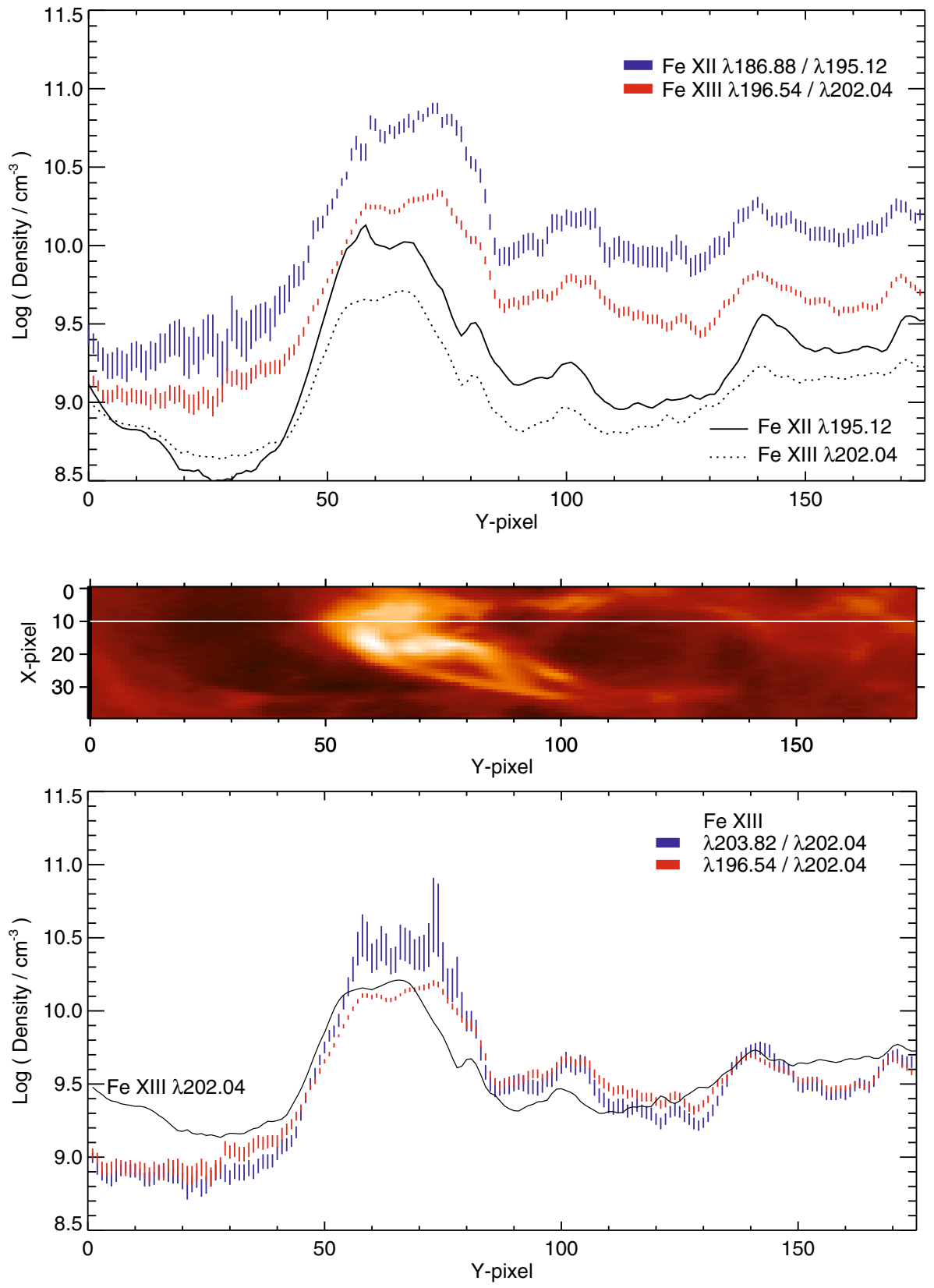

Fig. 19. A comparison of densities derived from the Fe XII $\lambda 186.88 / \lambda 195.12$ (blue lines) and Fe XIII $\lambda 196.64 / \lambda 203.82$ (red lines) ratios in the May 3 data set. The intensity curves from Fe XII $\lambda 195.12$ and Fe XIII $\lambda 202.04$ show the quantity $\log \left(10^{6} I\right)$ where $I$ is the line intensity in units erg $\mathrm{cm}^{-2} \mathrm{~s}^{-1} \mathrm{sr}^{-1}$.
Fig. 20. Exactly analogous plot to Fig. 18, except the Fe XIII densities are derived using the Keenan et al. (2007) atomic model. pattern, it is also clear that the velocity offsets discussed above for the May 6 data set are consistent with those for May 3, confirming the integrity of the EIS wavelength scale over time. An interesting effect in the velocity plots for May 3 is the loop-like structure visible for the red-shifted pixels and most clearly seen in the Fe XIII $\lambda 196.54-\lambda 202.04$ plot, but also partly seen in each of the other three plots. No explanation can be offered at this point for such an effect.

In summary, the plots shown in Fig. 21 give confidence in the quality of the Gaussian fits employed in the previous sections. In particular we see no evidence of line blending (beyond that already accounted for) that would lead to anomalous broadening or line shifts.

\section{Discussion}

Sections 6 and 7 compared the derived densities from the four Fe XII and Fe XIII line ratios and significant differences were seen between the two ions. In this section we consider whether these differences demonstrate real physical differences between the Fe XII and Fe XIII emitting regions, or whether atomic or instrumental effects could be responsible. Firstly we consider the pairs of ratios from each species.

Fe XII $\lambda 196.64 / \lambda 195.12$ systematically yields higher densities than $\lambda 186.88 / \lambda 195.12$ in both data sets. Section 6 identified a blending Fe VIII line as being partly responsible, but this does not explain the consistent discrepancies seen in the May 3 data set. The $\lambda 196.64 / \lambda 186.88$ ratio is actually relatively insensitive to density (a fact used in Appendix $C$ to help estimate the grating tilt), and Fig. 22 compares how the measured ratio values from the two data sets compare with the predictions from CHIANTI. The measured ratio values are plotted against the density derived from $\lambda 186.88 / \lambda 195.12$, with the predicted ratio variation with density from CHIANTI over-plotted. It is clearly seen that CHIANTI systematically under-estimates the measured ratios except for a small group of points in the May 3 data set. The shape of the gently sloping curve is reproduced quite well, except the high density points are a little further from 


\section{Fe XII}
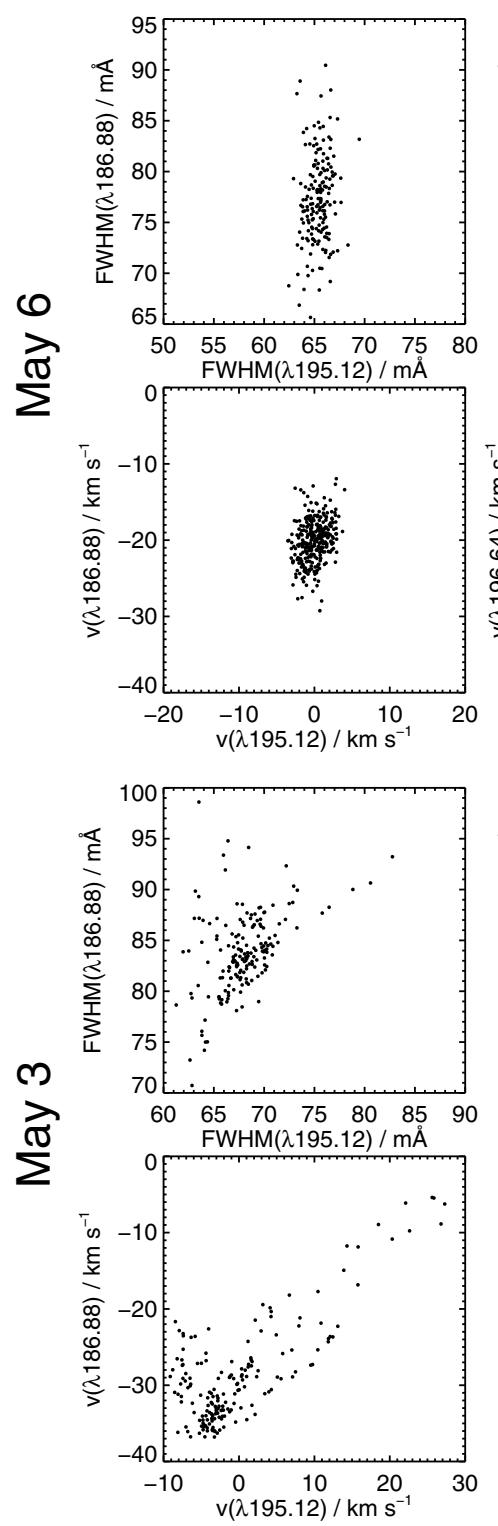
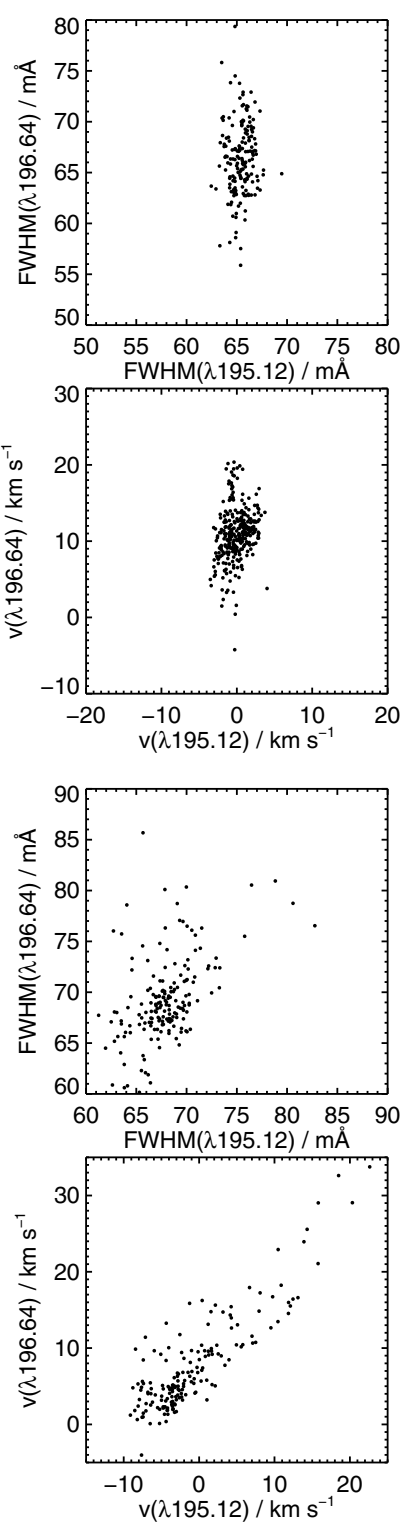

Fe XIII
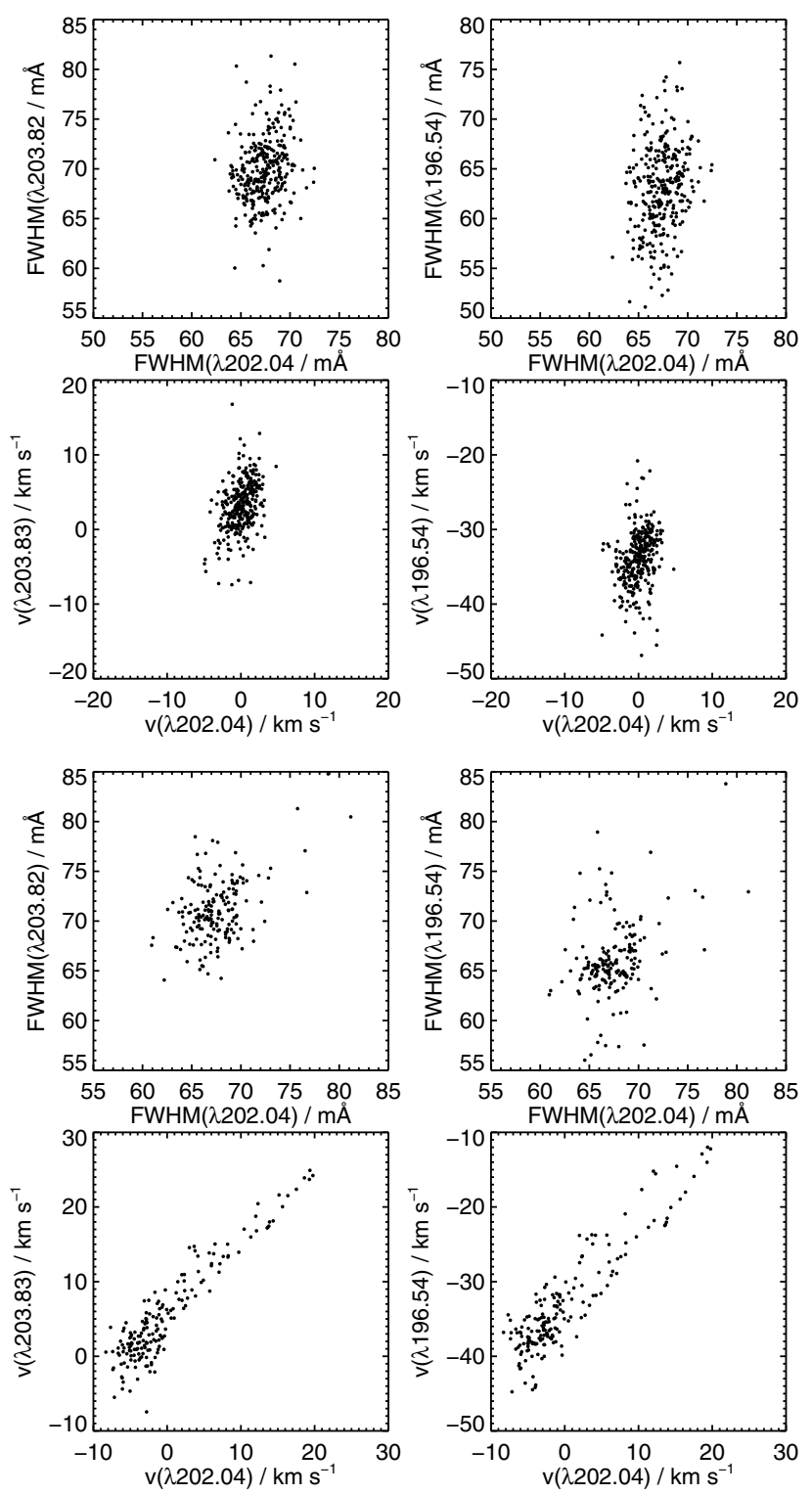

Fig. 21. Comparisons of line widths and velocities for the density diagnostic lines of Fe XII and Fe XIII from the May 6 and May 3 data sets. For Fe XII, $\lambda 195.12$ is used as the reference line, while for Fe XIII $\lambda 202.04$ is used. In each plot a dot indicates the value of FWHM or velocity from the two lines being compared at a particular spatial pixel along the data columns discussed in Sects. 6 and 7. The top two rows of plots show comparisons from May 6, while the bottom two rows show comparisons from May 3. The two left-most columns contain the comparisons for the Fe XII lines, and the two right-most columns the comparison for the Fe XIII lines.

the curve, explaining why the difference in derived densities is larger when the densities are high (Fig. 17). The effect of the blending Fe VIII line on $\lambda 196.64$ is clearly seen through the position of the small group of squares in the plot which denote spatial locations where Fe VIII is estimated to contribute more than $6 \%$ of the intensity of the measured line at $196.64 \AA$. An additional blending line could explain the systematic offset between the measured and predicted values of the $\lambda 196.64 / \lambda 186.88$ curve, but such a line would have to show very similar temperature and density behaviour to $\lambda 196.64$ and this is highly unlikely.

Based on the fact that $\lambda 186.88 / \lambda 195.12$ gives densities closer to Fe XIII than $\lambda 196.64 / \lambda 195.12$ we believe that the former ratio is thus more accurate. The discrepancy between observations and theory revealed in Fig. 22 is then due to CHIANTI underestimating the $\lambda 196.64 / \lambda 195.12$ ratio, however, a systematic error in the relative calibration of EIS of around $10-15 \%$ between wavelengths $186.88 \AA$ and $196.64 \AA$ can not be ruled out.

The discrepancy between the densities predicted by Fe XIII $\lambda 203.82 / \lambda 202.04$ and $\lambda 196.54 / \lambda 202.04$ only becomes significant at high densities and this clearly lies in a problem with the $\lambda 203.82 / \lambda 202.04$ ratio, our reason being that the density variation from $\lambda 196.54 / \lambda 202.04$ shown in Fig. 19 tracks the variation from Fe XII $\lambda 186.88 / \lambda 195.12$ very well. The densities from $\lambda 203.82 / \lambda 202.04$ increase anomalously fast compared to the three other ratios when the density is above $10^{10} \mathrm{~cm}^{-3}$. We can investigate this effect a little further by plotting the measured $\lambda 203.82 / \lambda 202.04$ ratio against the densities derived from $\lambda 196.54 / \lambda 202.04$, and comparing with the predicted ratio variation from CHIANTI (Fig. 23). It is seen that above $10^{10} \mathrm{~cm}^{-3}$ the measured ratio values are not actually far from the theoretical curve, but because the curve flattens at these densities the 


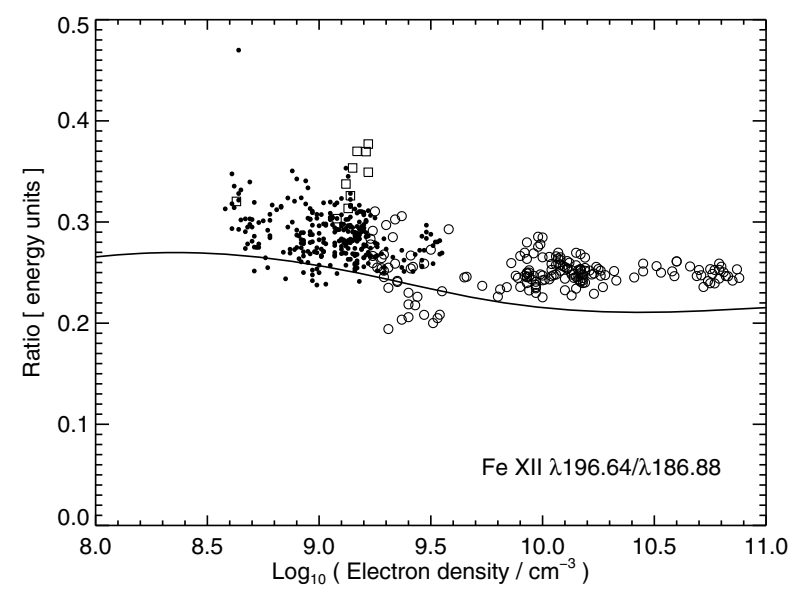

Fig. 22. A comparison of the measured variation of the Fe XII $\lambda 196.64 / \lambda 186.88$ intensity ratio with density compared to the predictions from the CHIANTI database. The measured densities are obtained from the Fe XII $\lambda 186.88 / \lambda 195.12$ ratio. Small filled circles denote measurements from the May 6 data set, larger open circles denote measurements from the May 3 data set. Points from the May 6 data set where Fe VIII is predicted to contribute $6 \%$ or more to the blended $\lambda 196.64$ line are denoted by open squares.

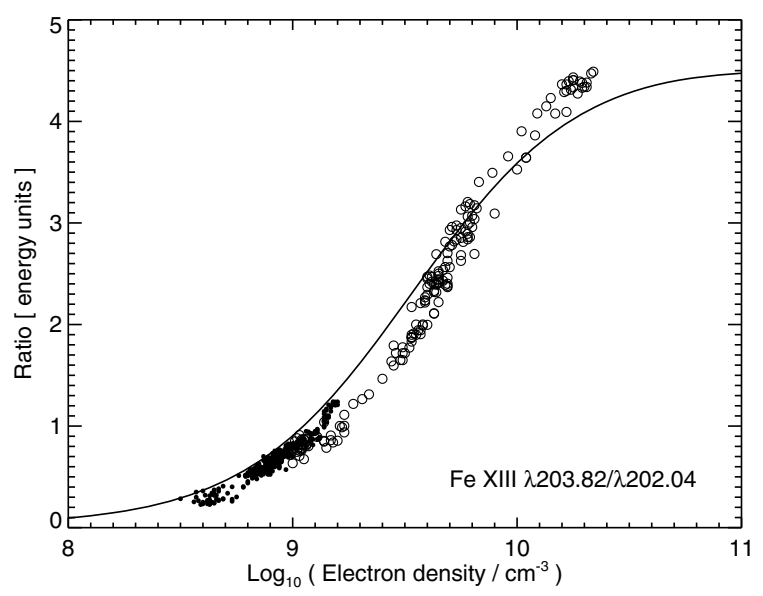

Fig. 23. A comparison of the measured variation of the Fe XIII $\lambda 203.82 / \lambda 202.04$ intensity ratio with density compared to the predictions from the CHIANTI database. The measured densities are obtained from the Fe XIII $\lambda 196.54 / \lambda 202.04$ ratio. Small filled circles denote measurements from the May 6 data set, larger open circles denote measurements from the May 3 data set.

measured ratios translate to high densities using the CHIANTI model. A discrepancy of a similar magnitude is seen around ratio values of 1.5 , but this translates to only a small difference in density due to the high gradient of the theoretical curve at this location. A simple remedy to the high density problem is if the high-density limit predicted by CHIANTI is increased from the current value of 4.5 to around 5.0. The Keenan et al. (2007) Fe XIII model predicts a high-density limit of 4.7 (Fig. 1) and so the densities derived from the May 3 data set are correspondingly lower in the high density region (Fig. 20). The highdensity limit for $\lambda 203.82 / \lambda 202.04$ will be a valuable test for future atomic calculations of Fe XIII.

Figures 14 and 19 showed the density comparisons between Fe XII and Fe XIII for what we consider to be the best diagnostics for each ion. The features in the density profiles generally match very well as might be expected for two ions formed at very similar temperatures, however the absolute density values differ, with Fe XII almost always giving higher densities than Fe XIII. Also striking in the May 6 data set (Fig. 14) is that the range of density variation of Fe XII is greater than that for Fe XIII.

Can the differences be due to a systematic error in the relative calibration of the EIS instrument? By artificially varying the instrument sensitivity at $202 \AA$ one finds that the densities from Fe XII $\lambda 186.88 / \lambda 195.12$ and Fe XIII $\lambda 196.54 / \lambda 202.04$ from the May 3 data set can be brought into line if the sensitivity at $202 \AA$ sensitivity is increased by $35 \%$ (i.e., the measured DN value at $202 \AA$ converts to a smaller line intensity, thus increasing the line ratio and the derived density). However such a large change would also mean that the sensitivity at the nearby wavelength of $203.8 \AA$ must also be significantly increased, which would then spoil the excellent agreement between the Fe XIII $\lambda 196.54 / \lambda 202.04$ and $\lambda 203.82 / \lambda 202.04$ ratios seen in Fig. 13. A detailed study of insensitive line ratios around 190-205 $\AA$ will be required to confirm that the EIS relative calibration is sound, but we believe a discrepancy of $35 \%$ is highly unlikely.

Ruling out a calibration problem, we can now ask: should Fe XII and Fe XIII actually yield the same density values? Even if the two source regions for the emitting lines do not have the same densities, an assumption of constant pressure would only lead to discrepancies of around 0.1 dex (although the higher density this implies for the cooler Fe XII ion is consistent with the measurements). A key issue to consider is whether the discrepancies could be caused through the existence of multiple density components along the line of sight: an implicit assumption when generating the plots is that the plasma observed has a fixed density for the line of sight corresponding to each pixel. This is generally not the case in the corona. Even if a coronal loop, for example, has a fixed density at the location being considered, the loop is sitting in a coronal background that could have a varying density with height (in the case of a hydrostatic equilibrium), or multiple densities due to a variety of loop like structures along the line of sight. The measured density then actually represents a weighted average of the different plasma components (e.g., Doschek 1984).

To look at this effect further, we consider the May 6 data set. Consider the spatial region around $Y$-pixel 150 where the Fe XII and Fe XIII densities become very similar. This region is seen in the upper panels of Figs. 12 and 13 to be free of loops and thus correspond to the background corona. In the nearby pixel regions 90-130 and 160-170 which are seen to correspond to active region loops, the discrepancy between the two ions sharply rises to $0.2-0.3$ dex. Is this discrepancy due to the background subtraction skewing the loop density measurements?

Figure 24 shows the densities derived from Fe XII $\lambda 186.88 / \lambda 195.12$ and Fe XIII $\lambda 203.82 / \lambda 202.04$ after a background contribution to the line intensities has been subtracted. For each line, the background intensity was calculated by averaging the intensities in $Y$-pixel region 132 to 143 . These background intensities were then subtracted from the intensities in the "loop" region ( $Y$-pixels 90-120), and the densities re-derived. Comparing with Fig. 14 the background subtractions have resulted in higher densities across the loop region by about 0.2 dex, but the discrepancy between Fe XII and Fe XIII has not been resolved. Considering the central part of the loop region ( $Y$-pixels 95-105) the average difference between Fe XII and Fe XIII was 0.28 dex before background subtraction, and becomes 0.27 dex after subtraction. In summary, then, background subtraction does not affect the Fe XII-Fe XIII discrepancy in this case. 


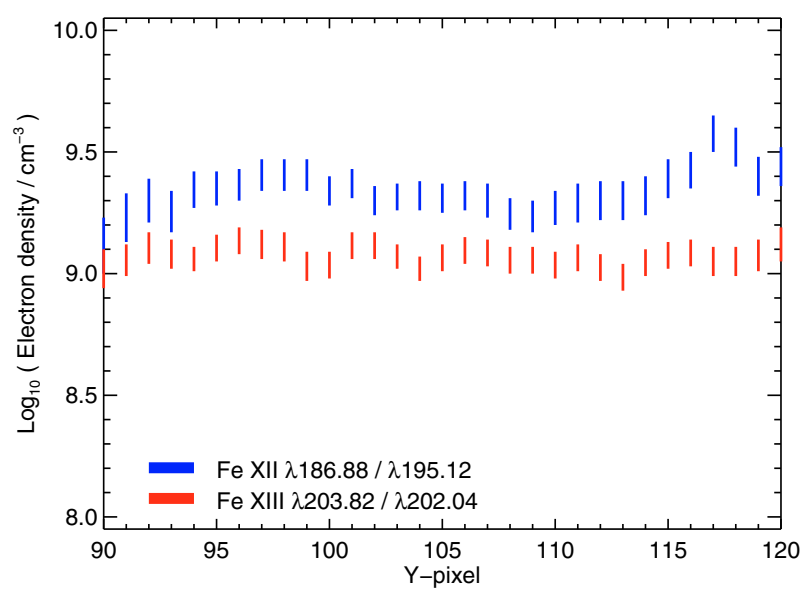

Fig. 24. A comparison of densities derived from Fe XII $\lambda 186.88 / \lambda 195.12$ and Fe XIII $\lambda 203.82 / \lambda 202.04$ for the loop bundle located at $Y$-pixels 90-120 in the May 6 data set. A background level has been subtracted from each of the line intensities, thus leading to different densities than those shown in Figs. 14.

The May 3 data set shows a much more complex plasma and so considerations of background subtraction on the density values are more difficult. However, the density in the $Y$-pixel region 60-80 is so high that the emission from this region will overwhelm any contribution from the background and thus we can speculate that it is a relatively "pure" measurement of the region's density. Despite this the discrepancy between the two ions' density values is around $0.4-0.5$ dex. It is difficult to envisage any physical circumstances that could result in such a discrepancy. We also note the discrepancy over the $Y$-pixel region $100-175$ is consistently at the $0.4-0.5$ dex level even though there is a wide range of plasma structures in this region (see the upper panels of Figs. 17 and 18). Therefore we believe these discrepancies are largely due to atomic physics parameters for the two ions, rather than physical properties of the plasma.

A further check that can be performed on the diagnostics is to use the derived densities to estimate the column depth of the emitting region at each pixel. Comparisons of column depths from the two ions with the observed sizes of solar structures potentially allow a statement to be made about which ion is yielding the most accurate density values. This issue will be discussed in a following paper.

\section{Conclusions}

An active region was observed by Hinode/EIS on 2007 May 3 and May 6, and coronal density values have been derived from emission line ratios of Fe XII and Fe XIII ratios that are the most sensitive in the EIS wavelength bands. Methods for automatically fitting the emission lines at each pixel in the spectral images have been described and line blending discussed. The derived densities are presented in Figs. 12-19 and the following conclusions are drawn.

- In active region data sets the precision of the density measurements from the four Fe XII and Fe XIII can be as high as $\pm 5 \%$ in individual spatial pixels - a considerable improvement over all previous solar spectrometers.

- The high precision of the measurements reveals significant discrepancies between the four ratios, thus implying the $a c$ curacy of the measurements is only around a factor 2 .
- For Fe XII, the $\lambda 196.64 / \lambda 195.12$ ratio always yields higher densities than $\lambda 186.88 / \lambda 195.12$ by up to 0.4 dex in $\log N_{\mathrm{e}}$. This is believed to be due to atomic data effects and/or instrumental effects, rather than a real physical effect.

- For Fe XIII, the $\lambda 203.82 / \lambda 202.04$ ratio is in very good agreement with $\lambda 196.64 / \lambda 202.04$ for densities less than $\log N_{\mathrm{e}}=$ 10.0. Above this density $\lambda 203.82 / \lambda 202.04$ yields higher densities which is most likely due to an inaccuracy in the theoretical high-density limit for the ratio.

- Comparing Fe XII and Fe XIII, the Fe XII densities are higher than the Fe XIII densities at almost all spatial locations, with the discrepancy being largest (up to $0.5 \mathrm{dex}$ ) at high densities. This is most likely due to the atomic data for the ions, but no indication can be given here on which ion's densities are most accurate.

- The May 6 data set shows a greater range of density variation for Fe XII than for Fe XIII which may be due to real physical differences for the Fe XII and Fe XIII emitting regions.

In addition to the above a number of instrumental and data analysis problems have been identified in this work

- The EIS spectra are tilted relative to the CCD's axis in the SW band with a slope of $-0.0792 \AA /$ pixel, an effect due to a slight misalignment of the grating relative to the CCD. This can have a significant effect on derived densities and must be corrected for if the emission lines are separated by more than a few angstroms.

- It is necessary to account for the Fe XII line at $195.18 \AA$ when interpreting the intensity of the strong Fe XII $\lambda 195.12$ line at densities $\geq 10^{10} \mathrm{~cm}^{-3}$.

- Fe VIII $\lambda 196.65$ contributes to Fe XII $\lambda 196.64$ in regions where Fe VIII emission is enhanced. The size of the contribution can be estimated by measuring Fe VIII $\lambda 194.66$, however the current CHIANTI model for Fe VIII appears to underestimate the contribution.

- Fe XII $\lambda 186.88$ is blended with S XI $\lambda 186.84$, but the contribution of this line is found to be mostly at the level of $\leq 10 \%$, and has only a small effect on derived Fe XII densities.

- The rest wavelengths contained in CHIANTI for some of the Fe XII and Fe XIII lines are not correct. We find new wavelengths of $196.518 \pm 0.003 \AA$ for Fe XIII $\lambda 196.54$, and $196.647 \pm 0.003 \AA$ for Fe XII $\lambda 196.64$.

The discrepancies between the densities derived from the different Fe XII and Fe XIII diagnostics are a serious impediment to using the densities for science analysis. A further study into the effects of the discrepancies on plasma column depths and filling factors will be presented in a following paper, which may shed light on which ion yields the most accurate density values. We believe the discrepancies are largely due to inaccurate atomic parameters for one or both of the ions and so new investigations into these parameters are urgently required in order that scientists may make best use of the high quality EIS spectra.

Acknowledgements. We acknowledge F.P. Keenan, G. Del Zanna and the anonymous referee for useful comments on the manuscript. Hinode is a Japanese mission developed and launched by ISAS/JAXA, with NAOJ as domestic partner and NASA and STFC (UK) as international partners. It is operated by these agencies in co-operation with ESA and NSC (Norway).

\section{Appendix A: An investigation of the Fe XII 195.12 blend}

The strong FeXII $\lambda 195.12$ line has been known to have a significant blending component due to the 

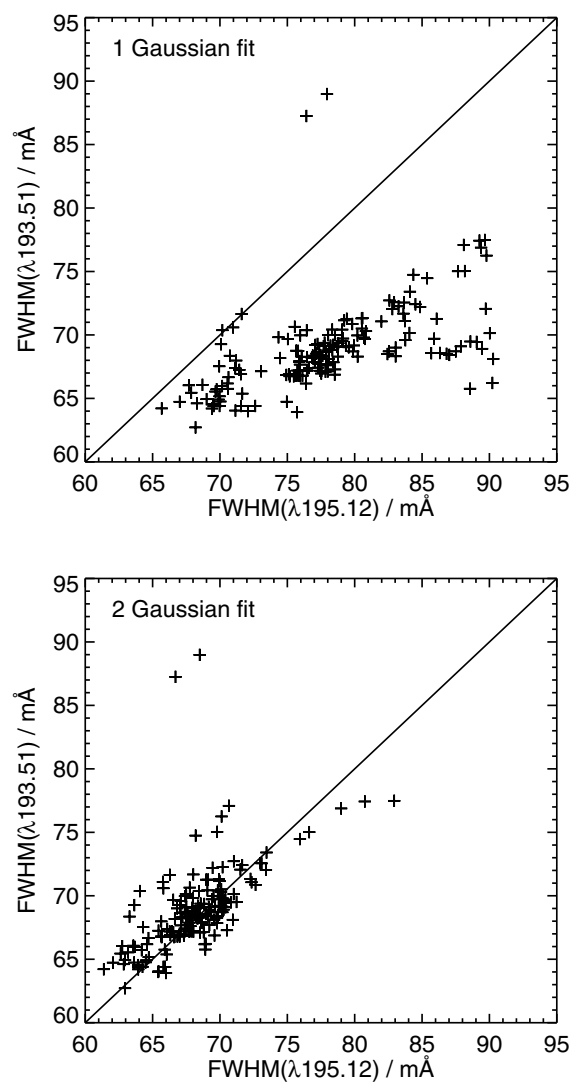

Fig. A.1. A comparison of the full widths at half maxima (FWHM) of Gaussian fits to the Fe XII $\lambda 195.12$ and $\lambda 193.51$ lines in the May 3 data set. The upper panel shows the results when a single Gaussian fit is applied to $\lambda 195.12$, the lower panel shows the results when a two Gaussian fit is applied in order to account for the blending $\lambda 195.18$ line.

$3 s^{2} 3 p^{3} \quad{ }^{2} D_{3 / 2}-3 s^{2} 3 p^{2}\left({ }^{1} \mathrm{D}\right) 3 d^{2} \quad D_{3 / 2}$ Fe XII transition since the work of Binello et al. (2001), who placed the latter transition at $195.13 \AA$. The wavelength was revised to $195.179 \AA$ by Del Zanna \& Mason (2005) based on unpublished work by Fawcett (Del Zanna, private communication 2008). In this section we investigate some of the properties of the fit parameters from our two Gaussian fits. We do this by making comparisons with a 1 Gaussian fit to the $195.12 \AA$ feature using the same wavelength pixels as marked in Fig. 6.

Firstly we compare the line widths of $\lambda 195.12$. It has been noted (Doschek et al. 2007a) that this line is broader than nearby Fe XII $\lambda 193.51$, and $\lambda 195.18$ was suggested as a possible cause of this (Young et al. 2007b). In Fig. A.1 we compare the widths of $\lambda 193.51$ and $\lambda 195.12$ measured from the May 3 data set when $\lambda 195.12$ is fit with a single Gaussian and when it is fit with a double Gaussian. The width of $\lambda 195.12$ is clearly seen to be larger than that of $\lambda 193.51$ with the one Gaussian fit, but they come into excellent agreement when a two Gaussian fit is used. This demonstrates that $\lambda 195.18$ is reponsible for the broader width of $\lambda 195.12$ noted by Doschek et al. (2007a).

A second issue related to $\lambda 195.18$ that can be investigated is the density sensitivity of the $\lambda 195.18 / \lambda 195.12$ ratio. Figure A. 2 shows the predicted variation of the ratio from CHIANTI where $\lambda 195.18$ is seen to become relatively stronger at high densities. Overplotted on this figure are observed ratios and densities from the May 6 and May 3 data sets. The densities are those obtained from the Fe XII $\lambda 186.88 / \lambda 195.12$ ratio. For densities $\geq 10^{10} \mathrm{~cm}^{-3}$ agreement is quite good, although there are a number of points significantly above the theoretical curve which are likely due to

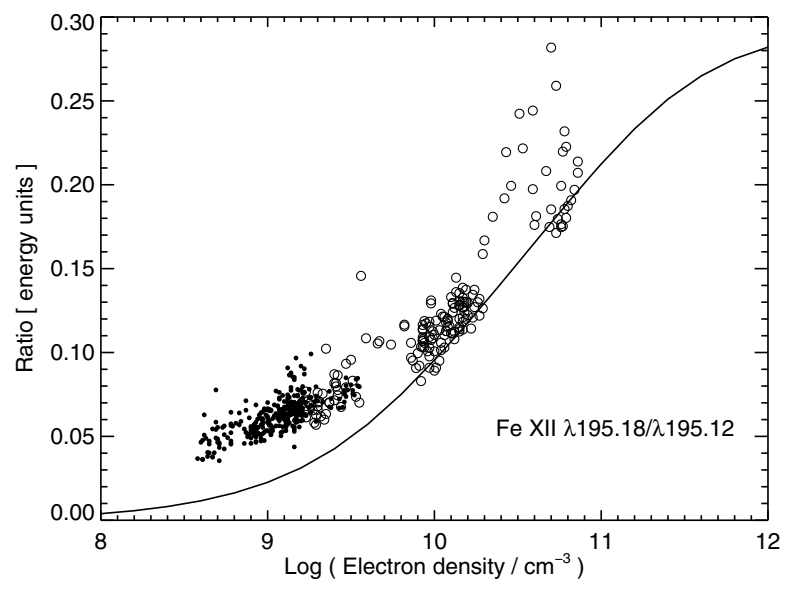

Fig. A.2. A comparison of the measured variation of the Fe XII $\lambda 195.18 / \lambda 195.12$ intensity ratio with density compared to the predictions from the CHIANTI database. The measured densities are obtained from the Fe XII $\lambda 186.88 / \lambda 195.12$ ratio. Small filled circles denote measurements from the May 6 data set, while larger, open circles denote measurements from the May 3 data set.

the $\lambda 195.18$ intensity being over-estimated by the two Gaussian fits.

At low densities, the observed ratios are all above the theoretical curve although the shape of the curve is reproduced quite well. This may be due to a systematic over-estimate of the $\lambda 195.18$ intensity in the fits due again to an unaccounted-for line.

\section{Appendix B: The Fe XII triplet 192.39, 193.51, 195.12}

The $3 s^{2} 3 p^{3}{ }^{4} S_{3 / 2}-3 s^{2} 3 p^{2}\left({ }^{3} \mathrm{P}\right) 3 d^{4} P_{5 / 2,3 / 2,1 / 2}$ transitions yield the three Fe XII transitions at 195.12, 193.51 and $192.39 \AA$, respectively. Their theoretical intensity ratios are almost completely insensitive to temperature and density and CHIANTI gives the values 1.0:0.67:0.32, respectively. All three lines are very strong in the EIS spectra (Young et al. 2007b) with $\lambda 195.12$ the strongest and so this line is most commonly observed with EIS. However, in active region observations $\lambda 195.12$ can be saturated making it difficult to use. In such circumstances it may be necessary to use $\lambda 192.39$ or $\lambda 193.51$ if they are observed with the EIS study as they are less likely to be saturated. In this section the relative intensities as measured from the May 3 and May 6 data sets are presented and compared with the CHIANTI predictions.

The ratios of the three lines will clearly be affected by blending with other species, and the contribution of $\lambda 195.18$ to the feature at $195.12 \AA$ was highlighted in Appendix A which demonstrated that $\lambda 195.18$ is responsible for the enhanced broadening of the $195.12 \AA$ feature when it is fit with a single Gaussian. Inspection of the line widths of $\lambda 192.39$ and $\lambda 193.51$ reveals no anomalies consistent with a lack of blending for these lines.

Figure B. 1 shows the $\lambda 192.39 / \lambda 195.12$ and $\lambda 193.51 / \lambda 195.12$ intensity ratios from the May 3 and May 6 data sets (lower and upper panels, respectively). Despite the large variation in density and intensity for the data sets, the ratios show excellent agreement with the CHIANTI theoretical values. The only discrepancy is that the $\lambda 193.51 / \lambda 195.12$ ratio in the May 6 data set is noticeably above the CHIANTI prediction and the May 3 values, and no explanation can be given for this effect. Another effect that can be seen in both ratios is that the measurements rise slightly as the $Y$-pixel increases. This can be explained if the instrument sensitivity is slightly different towards the top of the EIS slit than at the bottom, becoming more sensitive at short 

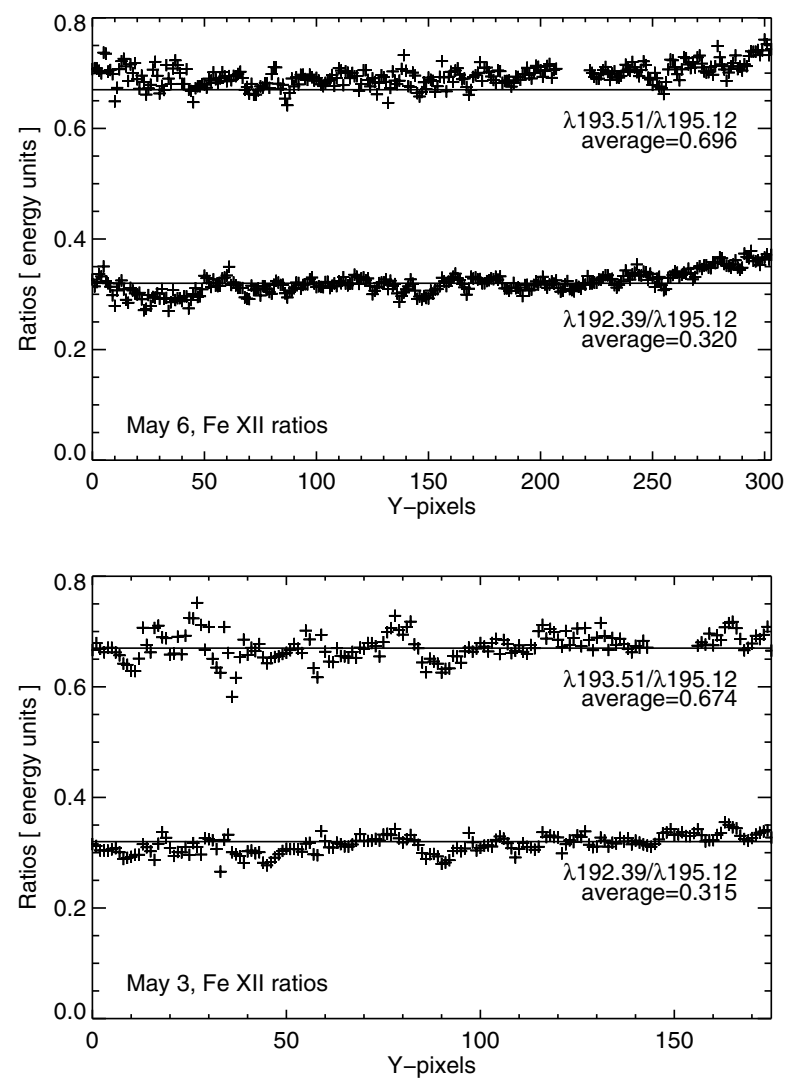

Fig. B.1. Comparison of measured Fe XII $\lambda 193.51 / \lambda 195.12$ and $\lambda 192.39 / \lambda 195.12$ intensity ratios against the predictions from CHIANTI from the May 6 (upper panel) and May 3 (lower panel) data sets. The solid horizontal lines in each panel show the line ratio values predicted from CHIANTI, and crosses denote the measured ratio values. The average of the measured values is shown for each of the ratios and data sets.

wavelengths at the top. The laboratory measurements performed by Seely et al. (2004) showed the shape of the multilayer coating reflectivity varying with the position of the light source in the aperture, and thus the Fe XII ratios could be revealing this effect.

In summary, though, the $\lambda 192.39 / \lambda 195.12$ and $\lambda 193.51 / \lambda 195.12$ ratios show excellent agreement with theoretical predictions and thus either $\lambda 192.39$ or $\lambda 193.51$ can be used in place of $\lambda 195.12$ when forming density diagnostics.

\section{Appendix C: Correcting for spatial offsets between emission lines}

The spectral image formed on the SW CCD is marginally tilted relative to the CCD axes such that the same spatial feature is observed to be slightly lower on the CCD in long wavelength lines compared to short wavelength lines. The effect is due to a misaligment of the grating relative to the CCD and we refer to it here as the "grating tilt". The best way to measure the tilt is to identify emission lines formed at the same temperature and which are density insensitive relative to each other. If a compact spatial feature is observed in such lines, then the offset can be estimated by co-aligning the images in the two lines. For the EIS SW band, however, there are few emission line pairs that are insensitive and span a wide enough wavelength range to be useful. In addition, most of the lines are formed in the corona where spatial features are more diffuse than in the transition region, making alignment more difficult. A further complication is that the tilt is small (a one spatial pixel offset over $12.6 \AA$ is found here), meaning for most line pair comparisons the offset is on the sub-pixel level.

For the present work the focus is on the density sensitive lines of Fe XII and Fe XIII which are found between 186.8 and $203.8 \AA$. We thus seek to identify the grating tilt over this region. We make use of the May 3 and May 6 data sets that have been used for the density analysis, and four line pairs are considered. Fe VIII images often show compact features that are very suitable for co-alignment (Young et al. 2007a), and the $\lambda 185.21$ and $\lambda 194.66$ lines are used here. They have a wide wavelength separation and only a small density sensitivity, however $\lambda 185.21$ is blended with a Ni XVI line and so care has to be taken to avoid comparing spatial features where there is clearly hot emission.

The remaining three ratios are all from Fe XII. Fe XII $\lambda 186.88 / \lambda 196.64$ involves two of the lines from the density analysis. CHIANTI predicts the intensity ratio to vary from 0.21 to 0.27 over the density range $10^{8}-10^{12} \mathrm{~cm}^{-3}$ and so the ratio is relatively insensitive to density. Fe XII $\lambda 192.39 / \lambda 195.12$ involves two very strong lines that are insensitive to density (Appendix B), however the wavelength separation is small. The last ratio is $\lambda 203.73 / \lambda 195.12$ which, although it shows significant density sensitivity, was deemed the best means of estimating the grating tilt out to the spectral feature at $203.8 \AA$. $\lambda 203.73$ is measured through a multi-Gaussian fit to Fe XIII $\lambda 203.82$ (Sect. 5.5).

The method for finding the spatial offset between two lines is as follows. Each of the lines was fit with a Gaussian as described in Sect. 5, yielding intensity images in each of the lines. A compact feature was then identified in particular image columns, and the intensity profiles along these columns extracted. One of the two columns was treated as a reference and, for the other, the intensity values were adjusted to simulate moving the image up and down on the detector. The intensity for this comparison column was re-calculated as

$I_{\text {new }}(i)= \begin{cases}(1-\alpha) I(i)+\alpha I(i+1) & \alpha>0 \\ (1-|\alpha|) I(i)+|\alpha| I(i-1) & \alpha<0\end{cases}$

where $\alpha$ is varied from -1 to +1 in 0.1 intervals. There are thus 21 columns to be compared with the reference column. From the reference column, the portion containing the compact spatial feature was extracted and compared with the 21 comparison columns to determine which gave the best spatial match. This was done by first normalising the extracted columns to the maximum value in the extracted region, and then calculating the quantity:

$w^{2}=\sum_{i}\left(I_{\text {ref }}^{*}(i)-I_{\text {new }}^{*}(i)\right)^{2}$

where $I_{\text {ref }}^{*}$ is the normalised intensity of the reference wavelength, and $I_{\text {new }}^{*}$ is the normalised comparison intensity (Eq. (C.1)). The best match to the reference column was determined to be the comparison column with the lowest $w^{2}$ value.

The May 6 data set had limited value for estimating the spatial offsets as Fe XII does not demonstrate any compact spatial features (e.g., the upper panel of Fig. 17). However, there is a bright, compact feature seen in Fe VIII (lower panel of Fig. 17). The offset from the $\lambda 185.21, \lambda 194.66$ comparison was found to be 0.7 pixels.

The May 3 data set showed a number of compact features at different temperatures however the Fe VIII comparison was compromised by the blending of $\lambda 185.21$ and so the offset from the 

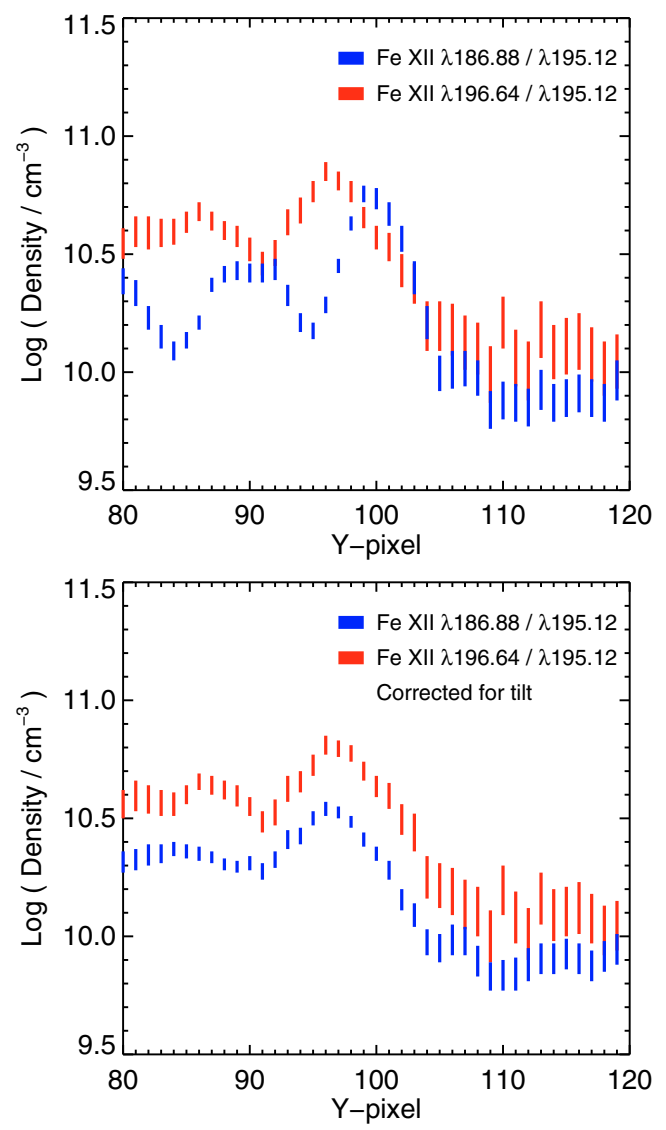

Fig. C.1. A comparison of densities obtained from the Fe XII $\lambda 186.88 / \lambda 195.12$ and $\lambda 196.64 / \lambda 195.12$ ratios measured from the May 3 data set. A section of a data column from $X$-pixel 25 (see upper panel of Fig. 17, for reference) is shown where is found a compact high density feature. The upper panel shows the densities obtained when the grating tilt is not corrected for, and the lower panel the results when the tilt is corrected for. Note that the discrepancy between the density peaks in the upper panel is resolved in the lower panel.

May 6 dataset was preferred. Comparing several features, the offset between Fe XII $\lambda 186.88$ and $\lambda 196.64$ was found to vary from 0.7 to 0.8 pixels - we assume 0.75 here. For $\lambda 195.12$ and $\lambda 203.72$ the offset was 0.8 pixels, and for $\lambda 192.39$ and $\lambda 195.12$ it was 0.2 pixels.

Assuming that the grating tilt is linear, the offset for a given wavelength is written as

$y=m(\lambda-195.12)$

where the offset is assumed to be zero at $195.12 \AA$. Computing the tilt from the four ratios and taking the average, we find $m=$ -0.0792 pixels/Å.

To illustrate the consequence of the grating tilt on the derived densities, we consider a small brightening that appears at $X$-pixel 25 and $Y$-pixel 98 in the May 3 data set (see Fig. 17). Figure C.1 compares the densities derived from the two Fe XII density diagnostics in the vicinity of this brightening. The top panel shows the densities when no offsets are applied, the bottom panel shows the densities when the offsets have been applied. It is clearly seen that the peaks in density around pixels 96-100 do not coincide in the top panel, but do coincide in the bottom panel. The discrepancy in the top panel is striking and greater than would be expected simply from the sub-pixel spatial offsets derived for the lines. This is because taking the ratios of lines amplifies the effect of the intensity offsets.
Finally we note that there is some uncertainty in deriving the grating tilt due to the difficulty of co-aligning data at the subpixel level. This thus represents a possible systematic error in the derived density measurements that will be greatest for those lines with a large wavelength separation. It may affect density comparisons between ratios for small spatial features, but not the broad trends highlighted in Sect. 9.

\section{References}

Aggarwal, K. M., \& Keenan, F. P. 2004, A\&A, 418, 371 Aggarwal, K. M., \& Keenan, F. P. 2005, A\&A, 429, 1117 Binello, A. M., Landi, E., Mason, H. E., Storey, P. J., \& Brosius, J. W. 2001, A\&A, 370, 1071

Brickhouse, N. S., Raymond, J. C., \& Smith, B. W. 1995, ApJS, 97, 551

Brooks, D. H., Warren, H. P., Ugarte-Urra, I., Matsuzaki, K., \& Williams, D. R. 2007, PASJ, 59, S691

Brosius, J. W., Davila, J. M., \& Thomas, R. J. 1998, ApJS, 119, 255

Brown, C. M., Hara, H., Kamio, S., et al. 2007, PASJ, 59, S865

Brown, C. M., Feldman, U., Seely, J. F., Korendyke, C. M., \& Hara, H. 2008, ApJS, 176, 511

Chifor, C., Young, P. R., Isobe, H., et al. 2008, A\&A, 481, L57

Culhane, J. L., Harra, L. K., James, A. M., et al. 2007, Sol. Phys., 243, 19

Del Zanna, G., \& Mason, H. E. 2003, A\&A, 406, 1089

Del Zanna, G., \& Mason, H. E. 2005, A\&A, 433, 731

Dere, K. P., \& Mason, H. E. 1981, in Solar Active Regions, ed. F. Q. Orrall (Boulder: Colorado Associated Univ. Press), 129

Dere, K. P., Landi, E., Mason, H. E., Monsignori-Fossi, B. C., \& Young, P. R. 1997, A\&AS, 125, 149

Dere, K. P., Landi, E., Young, P. R., \& Del Zanna, G. 2001, ApJS, 134, 331

Dere, K. P., Doschek, G. A., Mariska, J. T., et al. 2007, PASJ, 59, S721

Doschek, G. A. 1984, ApJ, 279, 446

Doschek, G. A., Warren, H. P., Laming, J. M., et al. 1997, ApJ, 482, L109 Doschek, G. A., Mariska, J. T., Warren, H. P., et al. 2007a, ApJ, 667, L109 Doschek, G. A., Mariska, J. T., Warren, H. P., et al. 2007b, PASJ, 59, S707 Fawcett, B. C., \& Mason, H. E. 1989, ADNDT, 43, 245

Feldman, U., Doschek, G. A., \& Cohen, L. 1983 ApJ, 273, 822

Flower, D. R. 1977, A\&A, 54, 163

Flower, D. R., \& Nussbaumer, H. 1974, A\&A, 31, 353

Flower, D. R., \& Pineau des Forêts, G. 1973, A\&A, 24, 181

Gupta, G. P., \& Tayal, S. S. 1998, ApJ, 506, 464

Imada, S., Hara, H., Watanabe, T., et al. 2007, PASJ, 59, S793

Keenan, F. P., Jess, D. B., Aggarwal, K. M., et al. 2007, MNRAS, 376, 205

Korendyke, C. M., Brown, C. M., Thomas, R. J., et al. 2006, Appl. Optics, 45, 8674

Jupén, C., Isler, R. C., \& Träbert 1993, MNRAS, 264, 627

Laming, J. M., Feldman, U., Schühle, U., et al. 1997, ApJ, 485, 911

Landi, E. 2002, A\&A, 382, 1106

Landi, E., Del Zanna, G., Young, P. R., et al. 2006, ApJS, 162, 261

Landman, D. A. 1975, A\&A, 43, 285

Landman, D. A. 1978, ApJ, 220, 366

Mariska, J. T. 1992, The Solar Transition Region (CUP)

Mariska, J. T., Warren, H. P., Ugarte-Urra, et al. 2007, PASJ, 59, S713

Neupert, W. M., Epstein, G. L., Thomas, R. J., \& Thompson, W. T. 1992, Sol. Phys., 137, 87

Penn, M. J., \& Kuhn, J. R. 1994, ApJ, 434, 807

Pike, C. D., \& Harrison, R. A. 2000, A\&A, 362, L21

Seely, J. F., Brown, C. M., Windt, D. L., Donguy, S., \& Kjornrattanawanich, B. 2004, Appl. Opt., 43, 1463

Storey, P. J., Del Zanna, G., Mason, H. E., Zeippen, \& C. J. 2005, A\&A, 433, 717

Tayal, S. S., Henry, R. J. W., Keenan, F. P., McCann, S. M., \& Widing, K. G. 1991, ApJ, 369, 567

Thompson, W. T., Haugan, S. V. H., \& Young, P. R. 1998, CDS Software Note No. 46, version 2, available from http://solar.bnsc.rl.ac.uk/ software/notes.shtml

Watanabe, T., Hara, H., Culhane, J. L., et al. 2007, PASJ, 59, S669

Young, P. R. 2004, A\&A, 417, 785

Young, P. R., \& Esser, R. 1999a, Solar Wind Nine, AIP Conf. Proc., 471, 273

Young, P. R., \& Esser, R. 1999b, Space Sci. Rev., 87, 345

Young, P. R., Landi, E., \& Thomas, R. J. 1998, A\&A, 329, 291

Young, P. R., Klimchuk, J. A., \& Mason, H. E. 1999, A\&A, 350, 286

Young, P. R., Del Zanna, G., Landi, E., et al. 2003, ApJS, 144, 135

Young, P. R., Del Zanna, G., Mason, H. E., et al. 2007a, PASJ, 59, S727

Young, P. R., Del Zanna, G., Mason, H. E., et al. 2007b, PASJ, 59, S857 